\title{
The XMM-Newton view of PG quasars
}

\section{X-ray continuum and absorption ${ }^{\star}$}

\author{
E. Piconcelli, E. Jimenez-Bailón, M. Guainazzi, N. Schartel, P. M. Rodríguez-Pascual, and M. Santos-Lleó \\ XMM-Newton Science Operation Center (ESAC), Apartado 50727, 28080 Madrid, Spain \\ e-mail: epiconce@xmm.vilspa.esa.es
}

Received 8 July 2004 / Accepted 26 October 2004

\begin{abstract}
We present results of a systematic analysis of the XMM-Newton spectra of 40 quasars (QSOs) $(z \leq 1.72)$ from the Palomar-Green (PG) Bright Quasar Survey sample $\left(M_{B}<-23\right)$. The sample includes 35 radio-quiet quasars (RQQs) and 5 radio-loud quasars (RLQs). The analysis of the spectra above $2 \mathrm{keV}$ reveals that the hard X-ray continuum emission can be modeled with a power law component with $\left\langle\Gamma_{2-12 \mathrm{keV}}\right\rangle=1.89 \pm 0.11$ and $\left\langle\Gamma_{2-12 \mathrm{keV}}\right\rangle=1.63_{-0.01}^{+0.02}$ for the RQQs and RLQs, respectively. Below $2 \mathrm{keV}$, a strong, broad excess is present in most QSO spectra. This feature has been fitted with four different models assuming several physical scenarios. All tested models (blackbody, multicolor blackbody, bremsstrahlung and power law) satisfactorily fitted the majority of the spectra. However, none of them is able to provide an adequate parameterization for the soft excess emission in all QSOs, indicating the absence of a universal shape for this spectral feature. An additional cold absorption component was required only in three sources. On the other hand, as recently pointed out by Porquet et al. (2004) for a smaller sample of PG QSOs, warm absorber features are present in 50\% of the QSO spectra in contrast with their rare occurrence $(\sim 5-10 \%)$ in previous studies. The XMM-Newton view of optically selected bright QSOs therefore suggests that there are no significant differences in the X-ray spectral properties compared with those of the low-luminosity Seyfert 1 galaxies. Properties of the $\mathrm{Fe} \mathrm{K} \alpha$ emission lines are presented in a companion paper.
\end{abstract}

Key words. galaxies: active - galaxies: nuclei - galaxies quasars: general - X-rays: galaxies

\section{Introduction}

Quasars (QSOs) emit a large amount of their emission in the X-ray band, where luminosities can reach $10^{46-47} \mathrm{erg} \mathrm{s}^{-1}$ (Zamorani et al. 1981). Variability studies indicate that the $\mathrm{X}$-rays originated in a region very close to the central object, probably an accreting supermassive black hole. Therefore, the analysis of the emission in this energy range provides strong constraints for models of the mechanism powering the QSOs. X-ray emission properties of QSOs have been largely studied during the last decades. Different samples of QSOs have been analyzed with previous X-ray satellites such as Einstein (Zamorani et al. 1981), EXOSAT (Comastri et al. 1992), Ginga (Lawson \& Turner 1997), ASCA (Cappi et al. 1997; George et al. 2000; Reeves \& Turner 2000), ROSAT (Schartel et al. 1997; Laor et al. 1997) and BeppoSAX (Mineo et al. 2000).

From these studies it emerges that the typical spectrum of a QSO in the hard ( $>2 \mathrm{keV}$ ) band is dominated by a power lawlike emission with a photon index $\Gamma \sim 2$. For Seyfert galaxies, the most popular physical scenario (Haardt \& Maraschi 1991) explains this hard spectral component as the emission

* Tables 2-8 and 10 are only available in electronic form at http://wWw.edpsciences.org originating in a hot corona placed above the accretion disk which comptonizes the UV-soft X-ray thermal emission from the disk and up-scatters it into the hard X-ray band. Part of the continuum emission is then reprocessed in the disk and/or in other nuclear physical components (i.e. the molecular torus, clouds) producing the typical reflection spectrum (George \& Fabian 1991). The most striking feature in the reflection spectrum is the fluorescent $\mathrm{Fe} \mathrm{K} \alpha$ emission line around $6.4 \mathrm{keV}$. This scenario has been extended to the QSOs, although the observational evidence for a reflection component in their X-ray spectra is poor. Signatures of the presence of large amounts of ionized and/or cold gas were also detected in the soft X-ray portion of QSO spectra (Cappi et al. 1997; George et al. 1997). It has also been reported that a fraction of QSOs present a gradual upturn in the emission emerging below $2-3 \mathrm{keV}$, the so-called soft excess (Comastri et al. 1992), whose nature is still debated.

Systematic analysis of QSOs with high spectral resolution provide a very useful tool for investigating their nature. In this paper we analyze a sample of QSOs observed with $X M M-N e w t o n$, which provides the highest throughput and sensitivity to date. We investigate the spectral characteristics of the objects in the sample and search for common features in order to elucidate the physical mechanisms responsible for the $\mathrm{X}$-ray emission. The sample includes both radio loud and radio 
quiet quasars (RLQs and RQQs, respectively). The classification is based on the strength of the radio emission relative to the optical. Many studies show systematic differences in the spectral properties in the X-ray band of both types of objects, such as for instance the slope of the power law (Laor et al. 1997; Reeves \& Turner 2000) which is evidence for a possible difference in the physical processes that give rise to the X-ray emission. Therefore the chosen sample provides useful information about possible differences between RLQs and RQQs. Results based on XMM-Newton observations of a smaller subsample of PG QSOs have recently been presented by Porquet et al. (2004; P04 hereafter). Thanks to the larger number of objects, our survey makes it possible to obtain a complete description of the X-ray spectral properties of optically selected QSOs based on a sounder statistical ground.

In Sect. 2 we present the general characteristics of the objects included in the sample, as well as the XMM-Newton observations and the reduction technique. We performed a systematic analysis of all objects assuming several physical scenarios for the emission of the QSO. The corresponding results are presented in Sect. 3, which also includes a detailed analysis of the most peculiar objects. In Sect. 4 we discuss observational constraints obtained by this study to the physical scenario for the $\mathrm{X}$-ray emission/absorption mechanisms at work in QSOs and we also compare them with previous similar works. Finally, the main results of this study are summarized in Sect. 5 .

\section{XMM-Newton observations}

\subsection{The sample}

The present sample is constituted by all Palomar-Green (PG) quasars (Schmidt \& Green 1983) with available EPIC observations in the XMM-Newton Science Archive (XSA) as of February 2004 plus PG $1001+054$, yielding a preliminary sample of 42 objects. Two QSOs $(0003+199$ and 1426+015) were excluded from the present study since all their EPIC exposures are affected by pile-up. Basic data for the final sample of $40 \mathrm{ob}-$ jects (i.e. name, coordinates, redshifts and the line-of-sight Galactic column density) are given in Table 1 . The redshifts span values from 0.036 to 1.718 . All but 5 QSOs $(0007+106$, $1100+772,1226+023,1309+355,1512+370$, i.e. $\sim 13 \%$ of the sample) are radio-quiet sources according to the definition proposed in Kellermann et al. (1994) i.e. exhibit a ratio of radio to optical emission $R_{\mathrm{L}} \leq 10$. It is worth noting that our sample includes all but two ${ }^{1}(1425+267$ and $1543+489)$ of the sources in the complete sample studied in Laor et al. (1997) (i.e. all the PG QSOs with $M_{B}<-23, z \leq 0.4$ and $\left.N_{\mathrm{H}}^{\mathrm{Gal}}<1.9 \times 10^{22} \mathrm{~cm}^{-2}\right)$.

\subsection{Data reduction}

The raw data from the EPIC instruments were processed using the standard Science Analysis System (SAS) v5.4.1 (Loiseau 2003) to produce the linearized event files for $p n$, MOS1 and MOS2. Only events with single and double patterns for

\footnotetext{
1 Both $1425+267$ and $1543+489$ have been observed by XMM-Newton but there were no public data.
}

the $p n$ (PATTERN $\leq 4)$ and single, double, triple and quadruple events for the MOS (PATTERN $\leq 12$ ), were used for the spectral analysis. The subsequent event selection was performed taking into account the most updated calibration files at the time of the reduction (September 2003). All known flickering and bad pixels were removed. Furthermore, periods of background flaring in the EPIC data were excluded using the method described in Piconcelli et al. (2004). Useful exposure times after cleaning are listed in Table 2 for each camera together with the date and the revolution of each reduced observation. In the case of $0844+349,1244+026$ and $1440+356$, the $p n$ observations are affected by pile-up, and therefore only the $M O S$ spectra were used for the analysis. On the other hand, for $1226+023$ and $1501+106$ only pn data were analyzed because of the impossibility to select a background region since the $M O S$ observations were carried out in small window mode. Source spectra were extracted from circular regions centered on the peak of the X-ray counts. Backgrounds were estimated in a source-free circle of equal radius close to the source on the same CCD. Appropriate response and ancillary files for both the $p n$ and the $M O S$ cameras were created using respectively the RMFGEN and ARFGEN tools in the SAS software. As the difference between the MOS1 and MOS2 response matrices is a few percent, we created a combined MOS spectrum and response matrix. The background-subtracted spectra for the $p n$ and combined $M O S$ cameras were then simultaneously fitted. According to the current calibration uncertainties we performed the spectral analysis in the $0.3-12 \mathrm{keV}$ band for $p n$ and in the $0.6-10 \mathrm{keV}$ band for the MOS cameras.

\section{Analysis of the spectra}

The source spectra were grouped such that each spectral bin contains at least 35 (or more in the case of the brightest sources) counts in order to apply the modified $\chi^{2}$ minimization technique and they were analyzed using XSPEC v.11.2 (Arnaud 1996). Galactic absorption (see Table 1) is implicitly included in all the spectral models presented hereafter. The photoelectric absorption cross sections of Morrison \& McCammon (1983) and the solar abundances of Anders \& Grevesse (1989) were used. The quoted errors refer to the $90 \%$ confidence level for one interesting parameter (i.e. $\Delta \chi^{2}=2.71$; Avni 1976). Throughout this paper we assume a flat $\Lambda C D M$ cosmology with $\left(\Omega_{\mathrm{M}}, \Omega_{\Lambda}\right)=(0.3,0.7)$ and a Hubble constant of $70 \mathrm{~km} \mathrm{~s}^{-1} \mathrm{Mpc}^{-1}$ (Bennett et al. 2003). All fit parameters are given in the quasar rest frame.

\subsection{Continuum emission above $2 \mathrm{keV}$}

A simple redshifted power law model was fitted to the hard $\mathrm{X}$-ray band, excluding the data below $2 \mathrm{keV}$ where additional spectral components like soft excess and absorbing features can heavily modify the primary source continuum. This fit turned out to be acceptable for most sources, with only five objects (i.e. $1211+143,1226+023,1411+442,1630+377$ and $2214+139)$ yielding an associated $\chi_{v}^{2}>1.2$. Some of these sources show a very flat spectrum with $\Gamma_{2-12}<1$ which suggests the presence of intrinsic absorption obscuring the primary 
Table 1. The sample.

\begin{tabular}{|c|c|c|c|c|c|}
\hline PG name & Other name & $\begin{array}{c}\text { RA } \\
(\mathrm{J} 2000)\end{array}$ & $\begin{array}{c}\text { Dec } \\
(\mathrm{J} 2000)\end{array}$ & $z$ & $\begin{array}{c}N_{\mathrm{H}}^{\mathrm{Gal}} \\
\left(10^{20} \mathrm{~cm}^{-2}\right)\end{array}$ \\
\hline $0007+106^{\star}$ & III Zw 2 & 001031.0 & +105830 & 0.089 & $6.09^{a}$ \\
\hline $0050+124$ & $\mathrm{I} Z \mathrm{Zw} 1$ & 005334.9 & +124136 & 0.061 & $4.99^{a}$ \\
\hline $0157+001$ & MKN 1014 & 015950.2 & +002341 & 0.163 & $2.46^{b}$ \\
\hline $0804+761$ & $1 \mathrm{H} 0758+762$ & 081058.6 & +760242 & 0.100 & $3.26^{c}$ \\
\hline $0844+349$ & TON 914 & 084742.4 & +344504 & 0.064 & $3.32^{c}$ \\
\hline $0947+396^{L}$ & & 095048.4 & +392650 & 0.206 & $1.92^{b}$ \\
\hline $0953+414^{L}$ & & 095652.1 & +411534 & 0.239 & $1.12^{c}$ \\
\hline $1001+054^{L}$ & & 100420.1 & +051300 & 0.161 & $1.88^{a}$ \\
\hline $1048+342^{L}$ & & 105143.8 & +335926 & 0.167 & $1.75^{b}$ \\
\hline $1100+772^{\star}$ & 3C 249.1 & 110413.7 & +765858 & 0.312 & $3.17^{e}$ \\
\hline $1114+445^{L}$ & & 111706.4 & +441333 & 0.144 & $1.93^{b}$ \\
\hline $1115+080$ & & 111816.0 & +074559 & 1.718 & $3.62^{b}$ \\
\hline $1115+407^{L}$ & & 111830.2 & +402553 & 0.154 & $1.74^{b}$ \\
\hline $1116+215^{L}$ & TON 1388 & 111908.6 & +211918 & 0.177 & $1.44^{a}$ \\
\hline $1202+281^{L}$ & & 120442.1 & +275411 & 0.165 & $1.72^{d}$ \\
\hline $1206+459$ & & 120858.0 & +454035 & 1.158 & $1.31^{d}$ \\
\hline $1211+143$ & & 121417.7 & +140313 & 0.081 & $2.76^{c}$ \\
\hline $1216+069^{L}$ & & 121920.9 & +063838 & 0.334 & $1.57^{c}$ \\
\hline $1226+023^{L \star}$ & $3 C 273$ & 122906.7 & +020308 & 0.158 & $1.89^{d}$ \\
\hline $1244+026$ & & 124635.3 & +022209 & 0.048 & $1.93^{a}$ \\
\hline $1307+085$ & & 130947.0 & +081948 & 0.155 & $2.11^{d}$ \\
\hline $1309+355^{L \star}$ & TON 1565 & 131217.8 & +351521 & 0.184 & $1.00^{b}$ \\
\hline $1322+659^{L}$ & & 132349.5 & +654148 & 0.168 & $1.89^{b}$ \\
\hline $1352+183^{L}$ & & 135435.6 & +180517 & 0.158 & $1.84^{a}$ \\
\hline $1402+261^{L}$ & & 140516.2 & +255535 & 0.164 & $1.42^{a}$ \\
\hline $1404+226$ & & 140621.8 & +222335 & 0.095 & $3.22^{a}$ \\
\hline $1407+265$ & & 140707.8 & +263230 & 0.940 & $1.38^{a}$ \\
\hline $1411+442^{L}$ & & 141348.3 & +440014 & 0.0896 & $1.05^{c}$ \\
\hline $1415+451^{L}$ & & 141701.24 & +445616 & 0.114 & $1.10^{e}$ \\
\hline $1427+480^{L}$ & & 142943.0 & +474726 & 0.221 & $1.69^{b}$ \\
\hline $1440+356^{L}$ & MKN 478 & 144207.4 & +352623 & 0.079 & $0.97^{b}$ \\
\hline $1444+407^{L}$ & & 144645.9 & +403505 & 0.267 & $1.27^{e}$ \\
\hline $1501+106$ & MKN 841 & 150401.2 & +102616 & 0.036 & $2.19^{b}$ \\
\hline $1512+370^{L \star}$ & $4 C+37.43$ & 151443.0 & +365050 & 0.371 & $1.36^{e}$ \\
\hline $1613+658$ & MKN 876 & 161357.2 & +654309 & 0.129 & $2.66^{a}$ \\
\hline $1626+554^{L}$ & & 162756.0 & +552231 & 0.133 & $1.55^{b}$ \\
\hline $1630+377$ & & 163201.2 & +373749 & 1.466 & $0.90^{a}$ \\
\hline $1634+706$ & & 163431.4 & +703134 & 1.334 & $5.74^{a}$ \\
\hline $2214+139$ & MKN 304 & 221711.5 & +141428 & 0.066 & $4.96^{d}$ \\
\hline $2302+029$ & & 230445.0 & +031146 & 1.044 & $5.27^{d}$ \\
\hline
\end{tabular}

${ }^{\star}$ Radio-loud objects. ${ }^{L}$ Objects included in Laor et al. (1997). References for $N_{\mathrm{H}}$ values: ${ }^{a}$ Elvis et al. (1989); ${ }^{b}$ Murphy et al. (1996); ${ }^{c}$ Lockman \& Savage (1995); ${ }^{d}$ Dickney \& Lockman (1990); ${ }^{e}$ Stark et al. (1992).

continuum. The resulting best-fit parameters are displayed in Table 3. Using the maximum likelihood technique (see Maccacaro et al. 1988), we have calculated the best simultaneous estimate of the average photon index of $\left\langle\Gamma_{2-12}\right\rangle$ and the intrinsic spread $\sigma\left(\Gamma_{2-12}\right)$. Figure 1 shows the $68 \%$, $90 \%$ and $99 \%$ confidence contours for the two parameters 


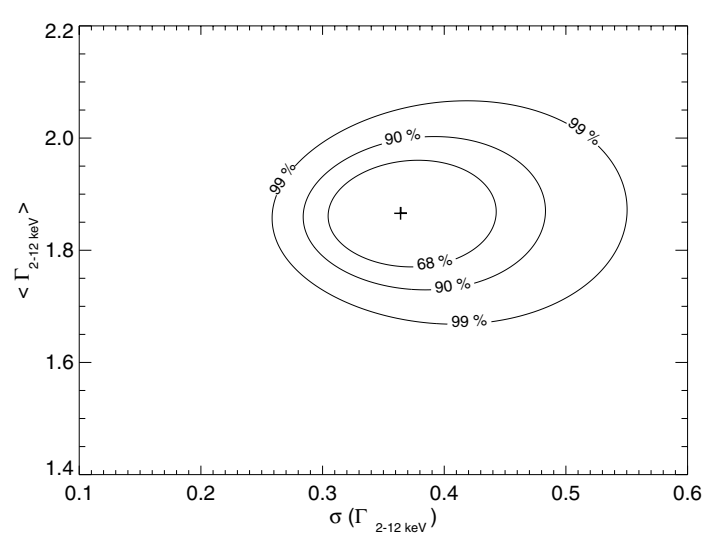

Fig. 1. Confidence contours for the simultaneous determination of the photon index $\left\langle\Gamma_{2-12}\right\rangle$ and the intrinsic dispersion $\sigma\left(\Gamma_{2-12}\right)$ together with the best-fit values found.

together with the best-fit values obtained, i.e. $\left\langle\Gamma_{2-12}\right\rangle=$ $1.87_{-0.10}^{+0.09}$ and $\sigma\left(\Gamma_{2-12}\right)=0.36_{-0.06}^{+0.08}$ (errors have been calculated using the $68 \%$ contour level).

Although the quality of the fit in the $2-12 \mathrm{keV}$ band is good for most quasars, the residuals show an excess around $6 \mathrm{keV}$ which suggests iron fluorescence emission. A detailed analysis of the $\mathrm{Fe} \mathrm{K} \alpha$ emission line is deferred to a second paper (see Jimenez-Bailon et al. 2004, Paper II hereafter).

\subsection{Systematic modeling of the $0.3-12 \mathrm{keV}$ continuum}

The extrapolation of the power law to energies lower than $2 \mathrm{keV}$ clearly revealed the presence of large deviations in all but one (i.e. 1206+459) spectra, with the most common residual feature being a smooth excess of soft X-ray flux. On the other hand, a handful of objects (i.e. 1001+054, 1114+445, $1115+080$ and 2214+139) exhibit a deep and sharp deficit in the $0.5-2 \mathrm{keV}$ band likely due to complex absorption.

\subsubsection{Power law fit}

The 0.3-12 keV spectrum of each source was fitted with a single power law model (PL) in order to provide an overall indication of the X-ray broad-band spectral shape $\left(\left\langle\Gamma_{0.3-12}\right\rangle=\right.$ $2.30 \pm 0.15$ and dispersion of $\left.\sigma\left(\Gamma_{0.3-12}\right)=0.60_{-0.09}^{+0.12}\right)$. The vast majority of $\chi_{v}^{2}$ values associated with this parameterization show that it yields a very poor description of the EPIC data (see Table 4 for the results). This is clearly a evident consequence of the inclusion in the fit of the $0.3-2 \mathrm{keV}$ range, which is dominated by the emission of the steeper "soft excess" component. Therefore, we fitted more complex models accounting for the soft excess (Sect. 3.2) and the absorption features (Sect. 3.4) if present in the spectrum. The presence of iron emission lines is investigated in Paper II.

\subsubsection{Soft excess modeling}

We accounted for the soft excess emission by means of four different two-component continua: (A) blackbody (BBODY in XSPEC) + power law; (B) multicolor blackbody (DISKBB in XSPEC) + power law; (C) bremsstrahlung (BREMSS in XSPEC) + power law; (D) power law + power law. Figure 2 shows some examples of source spectra fitted with the above models which can be considered representative of the different shapes of soft excess emission found in the sample.

Furthermore, photoelectric absorption edges were added to the fits whenever appropriate. We included these additional components by the $F-$ test criterion at a significance level $\geq 95 \%$. For the high- $z$ QSO $1206+459$, whose emission did not show any deviation from a simple power law continuum, we did not perform any further spectral fits. Eight sources (i.e. $0050+124,1001+054,1114+445,1115+080,1226+023$, $1404+226,1411+442$, and 2214+139) exhibited spectra more complex than a two-component continuum (plus absorption edge) and yielded a $\chi_{v}^{2} \geq 1.2$ for each of the tested models. They are not listed in the relative tables, but rather they are individually discussed in Sect. 3.4.

The best-fitting parameters resulting from the application of model A, B, C and D are shown in Table 5-8, respectively.

All objects for which we tested these models showed a statistically significant improvement in the goodness of fit upon the addition of a spectral component accounting for the soft excess. Using the maximum likelihood technique, we derived the best simultaneous estimate of the mean value and the intrinsic dispersion of the relevant parameter (i.e. $k T_{\mathrm{BB}}, k T_{\mathrm{MB}}, k T_{\mathrm{BS}}$ and $\Gamma_{\text {soft }}$ ) of each tested model. Figure 3 shows such best fit values as well as the corresponding $68 \%, 90 \%$ and $99 \%$ confidence contour levels. Finally, Table 9 lists the same values with the relative uncertainties given at the $68 \%$ confidence level for two relevant parameters.

\subsection{Spectral results for the complete subsample}

In this section we present the results of an analysis of the subsample of objects included in the Laor et al. (1997) study (marked with ${ }^{L}$ in Table 1). Laor et al. (1997) analyzed the ROSAT observations of 23 quasars from the Bright Quasar Survey with $z<0.4$ and $N_{\mathrm{H}}^{\mathrm{Gal}}<1.9 \times 10^{20} \mathrm{~cm}^{-2}$. We performed the analysis of 21 out of the 23 objects observed by Laor et al. (1997): there are no publicly available observations yet in the XMM-Newton archive for $1425+267$ and $1543+489$. The selection criteria for the Laor et al. (1997) sample only consider optical properties of the QSOs. Therefore the complete subsample is unbiased in terms of X-ray properties, and it is representative of the low-redshift, optically selected QSO population. Taking into account the results for the spectral analysis performed for the whole sample of quasars, we calculated the mean values of the relevant parameters of the models considered for the analysis: hard band power law and models $A, B, C$ and $D$. Table 9 shows the mean values and the dispersion of each parameter estimated using the maximum likelihood technique. Figure 4 shows the plots for the $68 \%, 90 \%$ and $99 \%$ confidence contours and the mean value and dispersion found for each spectral parameter. All the mean values of the different parameters found for the Laor subsample are fully compatible within the errors with the results for the whole sample. 

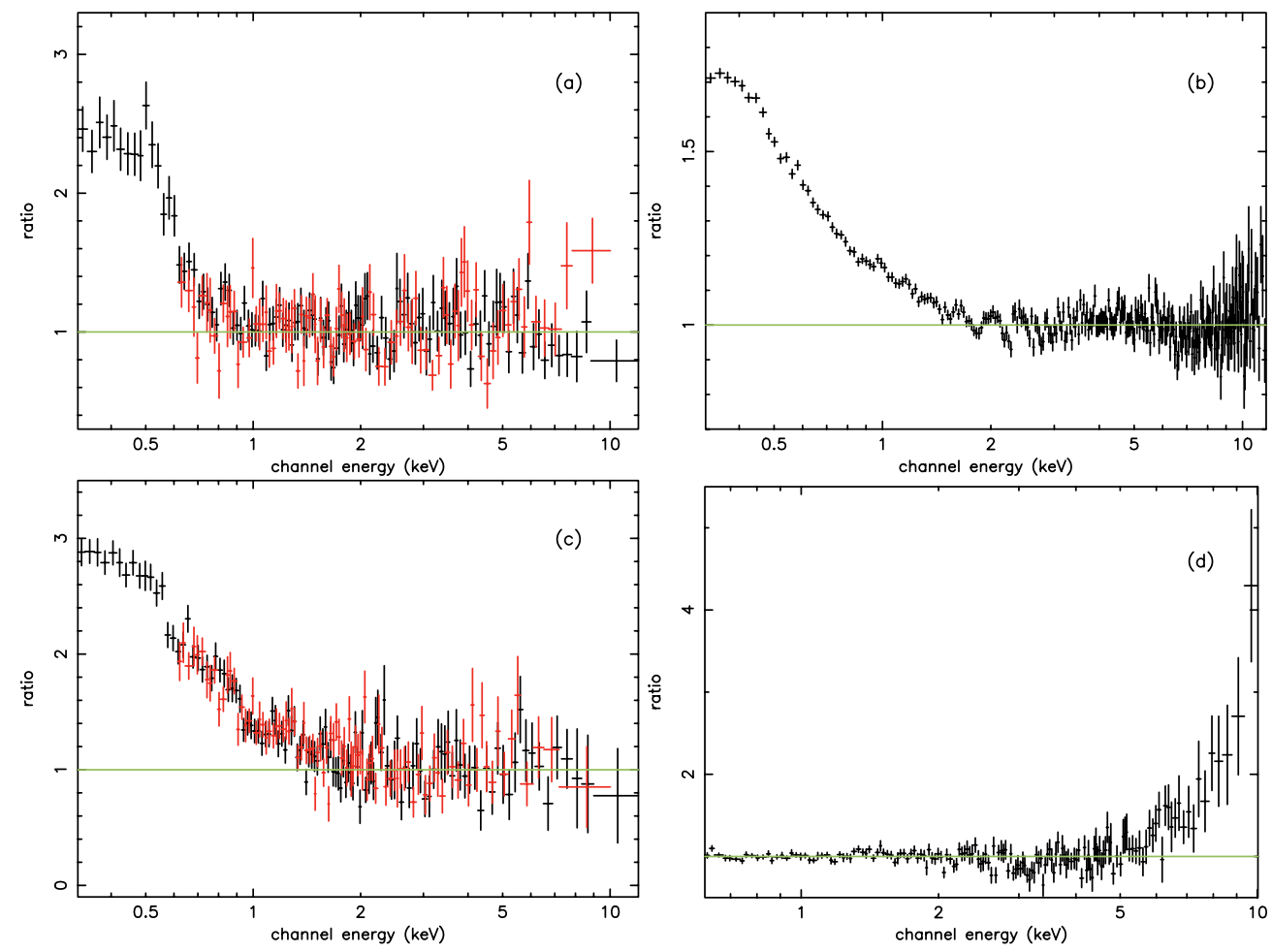

Fig. 2. The ratios (data/model) resulting from fitting a power law to the $2-12 \mathrm{keV} E P I C$ data ( $p n$ data in black, MOS in red) and extrapolating to lower energies in the case of the QSO (from top left) a) $1307+085(z=0.155)$ : the soft excess emission in this source proves to be best fitted with a blackbody (i.e. model A; see Table 10); b) 1226+023 ( $z=0.158)$ : note that the excess is broader than in $1307+085$ (the two QSOs have a similar $z$ ) which requires two blackbodies (i.e. model E; see Table 10) to be parametrized; c) $1352+183(z=0.158)$ for which model C (i.e. bremsstrahlung + power law) gives the best fit. Bottom right: d) the ratio resulted fitting with model A the EPIC data of $1440+356(z=0.079)$. Note the large residuals at high energies. This QSO is best fitted with a double power law model (i.e. model D; see Table 10).

\subsection{Best fit models}

The models tested to account for the soft excess can explain successfully the spectra of 30 out of the 40 sources. The spectra of the remaining nine sources have been analyzed separately (see below) while none of the two component models improved the quality of the fit for $1206+459$ likely because of the low signal-to-noise ratio of its spectrum. Even though the four models considered to explain the soft excess provided good fits $\left(\chi_{v}^{2}<1.2\right)$ for the majority of the sources, we found that for half of the sources, more complex parameterizations were required. Table 10 lists the best fit model as well as the resulting values of the spectral parameters for each quasar in the sample.

In the following we report on those sources for which the best fit model to the X-ray emission is more complex than a two-component parameterization. A consistent fraction of the QSOs in the sample, i.e. 13 out of 40 , required two thermal components to match their broad soft excess. Such sources have been fitted by the models labeled $\mathrm{E}$ and $\mathrm{H}$ in Table 10. In model E, both thermal components are modeled with a blackbody model. The mean temperatures obtained through the maximum likelihood method are $\left\langle k T_{\mathrm{BB}}\right\rangle=0.108 \pm 0.007 \mathrm{keV}$ for the lower, and $\left\langle k T_{\mathrm{BB}}\right\rangle=0.27_{-0.02}^{+0.03} \mathrm{keV}$ for higher temperature, while the dispersions are $\sigma=0.010_{-0.004}^{+0.007}$ and $\sigma=$ $0.041_{-0.017}^{+0.030}$, respectively. On the other hand, only one source, i.e. $1411+442$, required a blackbody plus a Raymond-Smith plasma (i.e. Model $\mathrm{H}$ in Table 10) to account for its soft excess (see below for further details on this source).

Spectral features due to absorption edges were reported in 16 quasars. The majority of them were found in the range $0.7-0.8 \mathrm{keV}$ : most these features are probably due to He-like oxygen and/or a blend of M-shell iron inner shell transitions (the so-called unresolved transition array or UTA; Behar et al. 2001). In three QSOs, i.e. $1115+080,1211+143,1226+023$, an edge was detected around 7.1-7.4 keV, implying reprocessing in ionized iron material. Interestingly, the spectrum of $1211+143$ shows three absorption edges, which are located, respectively, at $0.775_{-0.005}^{+0.011}, 0.967_{-0.009}^{+0.010}$ and $7.25_{-0.09}^{+0.14} \mathrm{keV}$. Since these absorption features are indicative of the presence of a warm absorber along the line-of-sight, we attempted a further fit using the model ABSORI in XSPEC (Done et al. 1992) to account for the ionized absorbing plasma in all the sources showing absorption edges. However, such parameterization did not significantly improve the goodness-of-fit statistic of any source expect for $1001+054,1114+445,1404+226$ and $2214+139$ (see below for more details on these sources), leaving the resulting gas parameters (i.e. $T, N_{\mathrm{H}}$ and $\xi^{2}$ ) basically unconstrained. The most likely explanation for this result is the

\footnotetext{
2 The ionization parameter $\xi$ is defined as $\xi=L / n r^{2}$, where $L$ is the isotropic luminosity of the ionizing source in the interval $5 \mathrm{eV}$ to $300 \mathrm{keV}, n$ is the number density of the warm plasma and $r$ is the distance between the latter and the central source.
} 



Fig. 3. Mean value and intrinsic dispersion of the relevant parameters of soft excess in models $\mathrm{A}\left(\left\langle k T_{\mathrm{BB}}\right\rangle\right), \mathrm{B}\left(\left\langle k T_{\mathrm{MB}}\right\rangle\right), \mathrm{C}\left(\left\langle k T_{\mathrm{BS}}\right\rangle\right)$ and D $\left(\left\langle\Gamma_{\mathrm{soft}}\right\rangle\right)$ together with 68\%, 90\% and 99\% confidence contours derived with the maximum likelihood method.

Table 9. Dispersion and mean value of the relevant parameters of the models tested for the sample and the Laor et al. (1997) subsample. See Sects. 3.2 and 3.3 for details. The mean values of the $k T$ in models A, B and C are given in units of keV.

\begin{tabular}{cccccc}
\hline \hline Model & Parameter & $\mu$ & $\sigma$ & $\mu_{\mathrm{LAOR}}$ & $\sigma_{\mathrm{LAOR}}$ \\
\hline Power law 2-12 keV & $\left\langle\Gamma_{2-12 \mathrm{keV}}\right\rangle$ & $1.87_{-0.10}^{+0.09}$ & $0.36_{-0.06}^{+0.08}$ & $1.82 \pm 0.13$ & $0.36_{-0.08}^{+0.11}$ \\
$\mathrm{~A}$ & $\left\langle k T_{\mathrm{BB}}\right\rangle$ & $0.136_{-0.008}^{+0.009}$ & $0.021_{-0.006}^{+0.010}$ & $0.133 \pm 0.010$ & $0.019_{-0.005}^{+0.009}$ \\
$\mathrm{~B}$ & $\left\langle k T_{\mathrm{MB}}\right\rangle$ & $0.157 \pm 0.008$ & $0.023_{-0.006}^{+0.008}$ & $0.160 \pm 0.010$ & $0.021_{-0.006}^{+0.010}$ \\
$\mathrm{C}$ & $\left\langle k T_{\mathrm{BS}}\right\rangle$ & $0.38 \pm 0.03$ & $0.08_{-0.02}^{+0.03}$ & $0.38 \pm 0.03$ & $0.05_{-0.02}^{+0.03}$ \\
$\mathrm{D}$ & $\left\langle\Gamma_{\text {soft }}\right\rangle$ & $2.73_{-0.11}^{+0.12}$ & $0.28_{-0.07}^{+0.11}$ & $2.80 \pm 0.08$ & $0.13_{-0.05}^{+0.08}$ \\
\hline
\end{tabular}

relatively limited photon statistics affecting the EPIC spectrum of these QSOs, which did not allow a more detailed description of the warm absorber than in terms of one (or more) edge(s).

Finally, in the following we report more details on the spectral analysis of the eight most peculiar sources in our sample, for which model A, B, C, and D gave an associate $\chi_{v}^{2} \geq 1.2$ (see Sect. 3.2).

$0050+124$ (I Zw 1). The XMM-Newton spectrum shows a complex soft $\mathrm{X}$-ray emission dominated by cold absorption. The observed data are best explained by a power law, $\Gamma=2.31 \pm 0.03$, accounting for the hard band and a double blackbody component, $k T_{1}=0.084 \pm 0.008 \mathrm{keV}$ and $k T_{2}=0.19_{-0.02}^{+0.03} \mathrm{keV}$. Our analysis reveals the presence of cold absorption modeled by the ZWABS model in XSPEC, $N_{\mathrm{H}}=9_{-1}^{+2} \times 10^{20} \mathrm{~cm}^{-2}$, and an absorption edge located at $0.65_{-0.02}^{+0.01} \mathrm{keV}$. These results are in agreement with a previous XMM-Newton data analysis by Gallo et al. (2004).

$1001+054$. We found that a power law modified by a warm absorber provides the best fit for this soft X-ray weak QSO. The resulting photon index is $\Gamma \sim 2$, and we derived a column density $N_{\mathrm{H}} \sim 2 \times 10^{23} \mathrm{~cm}^{-2}$ and an ionization parameter $\xi \sim 500 \mathrm{erg} \mathrm{cm}^{-2} \mathrm{~s}^{-1}$ for the absorbing gas. The spectrum of this QSO is discussed in detail in a separate paper (Schartel et al. 2004).

1114+445. An HST spectrum of this quasar revealed strong UV absorption lines (Mathur et al. 1998). In the X-ray band the presence of optically thin partially ionized material was detected (Laor et al. 1997; George et al. 1998). By applying a power law model to the $2-12 \mathrm{keV}$ data we obtained a flat photon index $\left(\Gamma_{2-12} \approx 1.4\right)$. Extrapolation down to lower energies shows a broad absorbing feature in the range $0.5-1 \mathrm{keV}$. The broad band X-ray continuum of this QSO is well described by a power law with $\Gamma \approx 1.85$ modified by two ionized absorption components (ABSORI in XSPEC) plus a blackbody component accounting for the soft excess (see Table 10 for the best fit parameters). The presence of the second "warm" component yielded an improvement in the fit at $>99.9 \%$ confidence level (e.g. Fig. 5). XMM-Newton has therefore clearly revealed, for the first time in this QSO, a multi-zone ionized absorber similar to what we found for $2214+139$ (see below; Piconcelli et al. 2004). We also reported the presence of a significant $\left(P_{F}>99.9 \%\right)$ fluorescence iron emission line with a narrow 


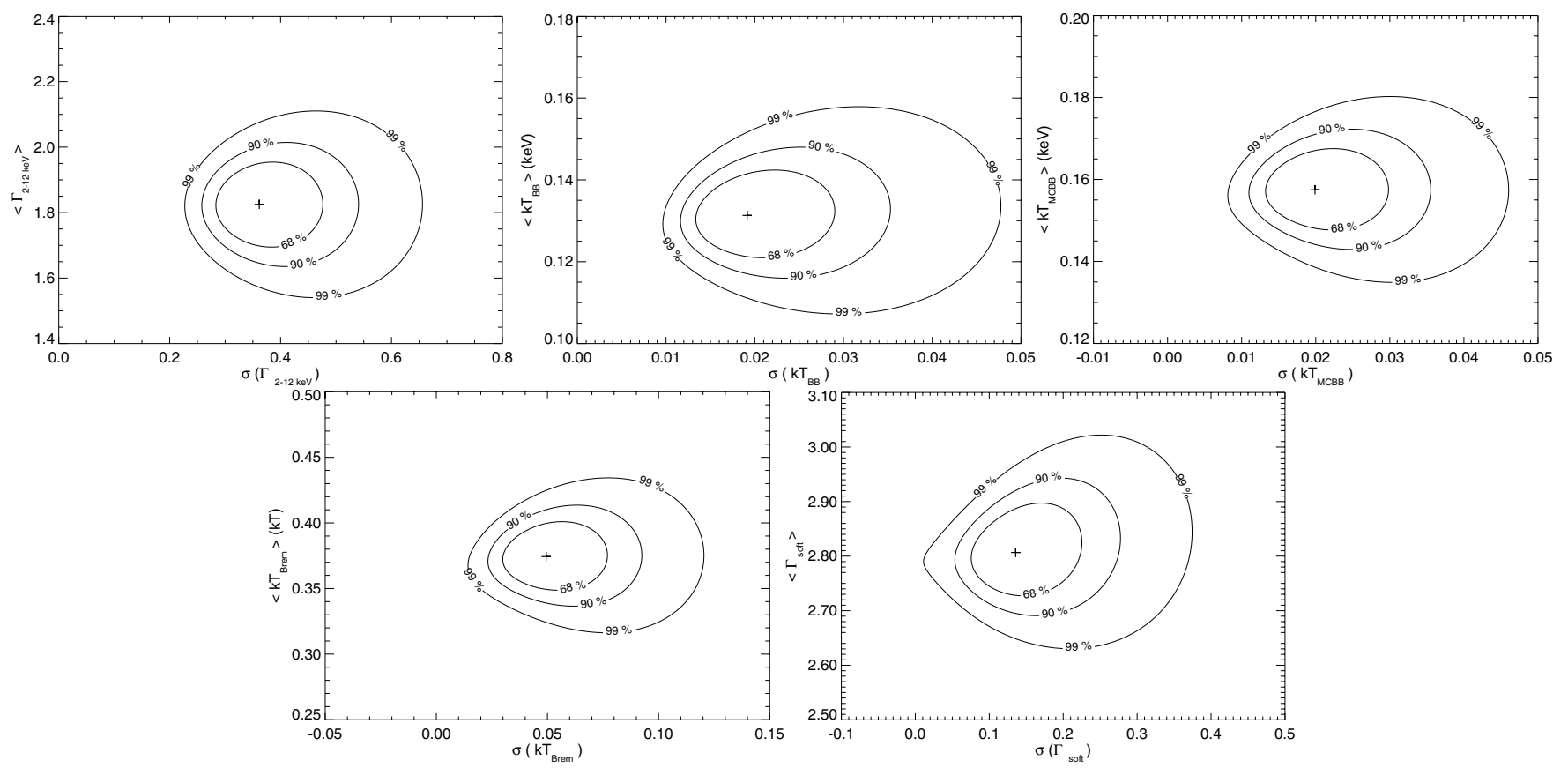

Fig. 4. Mean value and intrinsic dispersion of the relevant parameters for tested models applied to the Laor et al. (1997) quasars together with $68 \%, 90 \%$ and $99 \%$ confidence contours derived with the maximum likelihood method.

profile at $6.45_{-0.08}^{+0.02} \mathrm{keV}$ (e.g. Paper II). On the other hand, we did not find any evidence of a Fe K-shell absorption edge as detected at $\approx 7.25 \mathrm{keV}$ with a $\tau=0.35_{-0.29}^{+0.38}$ in the $A S C A$ spectrum by George et al. (1998). Our results agree with those recently published by Ashton et al. (2004) from an independent analysis of the same XMM-Newton data. They also detected a two-phase warm absorber even if they found a best fit value of the ionization parameter for the hotter-phase component of the warm absorber larger than ours (and, consequently, also a larger column density).

1115+080. XMM-Newton data of this BAL quasar have also been analyzed by Chartas et al. (2003). This study revealed an absorbed soft energy spectrum. Even though Chartas et al. (2003) suggested that the soft spectrum is best fitted by an ionized absorber, our study found that a neutral absorption model cannot be ruled out. Our analysis indicates that the best fit model for the observed spectrum consists of a power law modified by cold absorption and two absorption edges at $7.1_{-0.4}^{+0.3} \mathrm{keV}$ and $9.5 \pm 0.3 \mathrm{keV}$ (see Table 10 for further details). Taking into account the Chartas et al. (2003) results, we have tried to explain the soft energy absorption by an ionized absorber using the ABSORI model in XSPEC. The value found for the ionization parameter $(\xi)$ is extremely low and therefore the model does not differ from the neutral absorption one.

$1226+023$ (3C 273) The main feature in the $p n$ spectrum of this well-known radio-loud QSO is the broad soft excess. A simple two-component model indeed fails to adequately describe its $0.3-12 \mathrm{keV}$ emission (see Sect 3.2 and Fig. 2). Our best fit includes two blackbody components (with $k T_{\mathrm{BB}, 1} \approx 0.1$ and $k T_{\mathrm{BB}, 2} \approx 0.24 \mathrm{keV}$, respectively) and a power law with a quite flat photon index $(\Gamma=1.60 \pm 0.01)$. This result confirms the finding by Page et al. (2004a), based on the same
XMM-Newton observation. We also detected the significant (at $>99.9 \%$ confidence level) presence of an absorption edge at $7.4_{-0.2}^{+0.1} \mathrm{keV}$ and with an optical depth of $\tau=0.09_{-0.03}^{+0.02}$. Such an energy centroid suggests that the absorbing material is weakly ionized (Fe V-XV). The most likely origin for this edge feature is via reflection in optically thick matter, as an origin in a line-of-sight plasma is not supported by the $R G S$ data analysis results published in Page et al. (2004a), which did not report any absorption features apart from a OVII He $\alpha$ due to warm gas in the local intergalactic medium. Consequently, we also tried to fit the data with a model including a Compton reflection component (PEXRAV in XSPEC); however this model yielded no statistical improvement with respect to the best fit model listed in Table 10. The upper limit for the covering factor of the material irradiated by the X-ray source is $R=\Omega / 2 \pi=0.4$. On the other hand, no Fe K $\alpha$ emission line (i.e. another hallmark of reprocessing in an accretion disk) was detected, and the upper limit on the equivalent width for a narrow line at $6.4 \mathrm{keV}$ was found to be $6 \mathrm{eV}$. Finally, Page et al. (2004a) reported evidence for a weak broad Fe line using ten co-added EPIC observations. However, this line has not been detected in our data even when the absorption edge has been removed from the fitting model.

$1404+226$. This narrow line QSO was observed by ROSAT and ASCA. Ulrich et al. (1999) reported a very steep soft X-ray continuum, and evidence of a complex ionized absorber. In particular, a peculiar absorption feature around $1 \mathrm{keV}$ led these authors to claim deviations from solar abundances in this source. Our results confirm the spectral complexity of 1404+226: we have found a best fit model consisting of a blackbody with $k T=0.144_{-0.003}^{+0.002} \mathrm{keV}$ and a steep $\left(\Gamma=2.34_{-0.37}^{+0.35}\right)$ power law modified by ionized absorption. For the gas we derive a column density $N_{\mathrm{H}} \approx 1.4 \times 10^{22} \mathrm{~cm}^{-2}$ and an ionization 

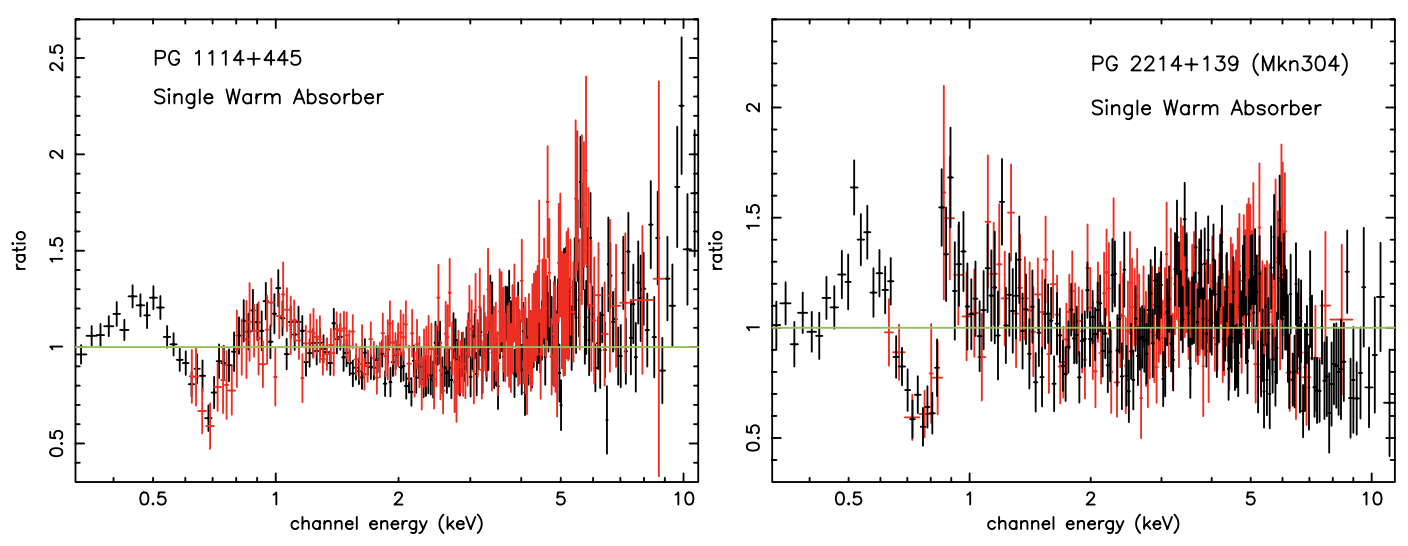

Fig. 5. The ratios between the EPIC data ( $p n$ data in black, MOS in red) and a fit with single warm absorber model for $1114+445$ (left) and $2214+139$ (right): the large residuals below $1 \mathrm{keV}$ strongly suggest the presence of a complex "multi-component" warm absorber. Note also the residuals around 5-6 keV which indicate $\mathrm{Fe} \mathrm{K} \alpha$ fluorescence emission (e.g. Paper II).

parameter $\xi=44_{-26}^{+123} \mathrm{erg} \mathrm{cm}^{-2} \mathrm{~s}^{-1}$. No evidence of elemental abundances different from solar values has been found. Finally, no significant fluorescence iron emission line has been detected.

$1411+442$. This BAL QSO is X-ray faint $\left(F_{0.3-12} \approx 7 \times\right.$ $10^{-13} \mathrm{erg} \mathrm{cm}^{-2} \mathrm{~s}^{-1}$ ). The $2-10 \mathrm{keV}$ spectrum is extremely flat with a $\Gamma_{2-12} \approx 0.4$ and a clear excess around $6 \mathrm{keV}$. The addition of a line with a narrow Gaussian profile to parameterize the $\mathrm{Fe} \mathrm{K} \alpha$ emission feature produced a very significant (at 99.8\% confidence level) improvement in the fit. The centroid $\left(E_{\mathrm{K} \alpha}=6.43_{-0.13}^{+0.05} \mathrm{keV}\right)$ and narrowness of the line suggest a likely origin from neutral material located far away from the innermost regions of the accretion disk.

The best fit model requires a power law with partialcovering (ZPCF in XSPEC) and a Raymond-Smith plasma component (the latter significant at $>99.9 \%$ confidence level). For the absorbing gas we obtained a column density $N_{\mathrm{H}} \approx 2.3 \times$ $10^{23} \mathrm{~cm}^{-2}$ and a covering fraction of $96 \%$. The plasma temperature and the photon index of the power law were found to be $k T=0.15 \pm 0.03 \mathrm{keV}$ and $\Gamma=2.3 \pm 0.2$, respectively.

The limited statistics did not allow us to test more complex fitting models aimed, for instance, at checking the presence of a warm absorber in this source, as observed in other AGNs with prominent absorption lines in the UV spectrum (e.g. Crenshaw et al. 2003; Monier et al. 2001; Piconcelli et al. 2004). This EPIC observation has also been analyzed by Brinkmann et al. (2004a) who reported results consistent with ours.

$2214+139$ (MKN 304). Our analysis matches well with previous results based on Einstein and ROSAT observations, which reported a very flat continuum for this source, and suggested the likely existence of heavy absorption. The $X M M$-Newton data reveal a complex spectrum, dominated by strong obscuration due to ionized gas (see Fig. 5). With an $N_{\mathrm{H}} \approx 10^{23} \mathrm{~cm}^{-2}$, it is one of the ionized absorbers with the highest column density seen so far by XMM-Newton and Chandra. A two-component warm gas provides an excellent description of this absorbing plasma. From the spectral analysis we derived $\xi^{\mathrm{T5}}=89.3_{-12.0}^{+13.9} \mathrm{erg} \mathrm{cm}^{-2} \mathrm{~s}^{-1}$ and $\xi^{\mathrm{T} 4}=5.9_{-0.9}^{+2.4} \mathrm{erg} \mathrm{cm}^{-2} \mathrm{~s}^{-1}$ for the hot (i.e. with $T=1.5 \times 10^{5} \mathrm{~K}$ ) and the cold (i.e. with $\left.T=3 \times 10^{4} \mathrm{~K}\right)$ component, respectively. In addition to a narrow $\mathrm{Fe} \mathrm{K} \alpha$ emission line at $\sim 6.4 \mathrm{keV}$, another emission line feature was significantly detected at $0.57 \mathrm{keV}$, likely due to the helium-like oxygen triplet (perhaps originating in the warm absorber itself). We also reported the presence of a weak soft excess component which can be interpreted as partial covering or scattered emission from the ionized outflowing plasma. For the complete and detailed presentation and discussion of these results see Piconcelli et al. (2004).

\subsection{Fluxes and luminosities}

Fluxes in the soft $(0.5-2 \mathrm{keV})$ and hard $(2-10 \mathrm{keV})$ energy band assuming the best fit model for each quasar as in Table 10 are listed in Table 11, together with the corresponding luminosities corrected for both the Galactic absorption and (if present) the additional intrinsic warm/neutral absorber column densities. The hard (soft) X-ray fluxes range from $\sim 0.1(0.1)$ to $\sim 80(45) \times 10^{-12} \mathrm{erg} \mathrm{cm}^{-2} \mathrm{~s}^{-1}$. With a $0.5-10 \mathrm{keV} \mathrm{X-ray}$ luminosity of $\sim 10^{43} \mathrm{erg} \mathrm{s}^{-1}, 1001+054$ is the faintest object in the sample. The most luminous quasar in the hard (soft) band is $1226+023(1407+265)$ with a luminosity of $\sim 50 \times$ $10^{44} \mathrm{erg} \mathrm{s}^{-1}$. In the last two columns of Table 11 the ratios between the strength of the soft excess and the high energy power law component in the $0.5-2$ and $0.5-10 \mathrm{keV}$ band are listed.

\subsection{Correlations among spectral parameters and luminosity}

We performed Spearman-rank correlation tests. This test calculates how well a linear equation describes the relationship between two variables by means of the rank correlation coefficient $\left(r_{\mathrm{s}}\right)$ and the probability $\left(P_{\mathrm{s}}\right)$. Positive(negative) values of $r_{\mathrm{s}}$ indicate that the two quantities are (anti-)correlated, while $P_{\mathrm{s}}$ gives the significance level of such a correlation. In particular, we checked the type and strength of the relationship among the representative parameter of each model (i.e. $k T_{\mathrm{BB}}, k T_{\text {DiskBB }}$, $k T_{\text {Brem }}, \Gamma_{\text {Soft }} ;$ see Sect. 3.2) and $L_{0.5-2 \mathrm{keV}}, L_{2-10 \mathrm{keV}}, R_{\mathrm{S} / \mathrm{P}}^{0.5-2}$ and $R_{\mathrm{S} / \mathrm{P}}^{0.5-10}$ and $\Gamma_{2-12}$ (see Tables 11 and 3). The corresponding results are listed in Table 12. 
Table 11. Fluxes and absorption-corrected luminosities calculated assuming the best fit model. The last two columns list the ratio between the strength of the soft excess and the high energy power law component in the $0.5-2$ and $0.5-10 \mathrm{keV}$ band, respectively.

\begin{tabular}{|c|c|c|c|c|c|c|}
\hline PG Name & $\begin{array}{c}F_{0.5-2 \mathrm{keV}} \\
\left(10^{-12} \mathrm{erg} \mathrm{cm}^{2} \mathrm{~s}^{-1}\right)\end{array}$ & $\begin{array}{c}F_{2-10 \mathrm{keV}} \\
\left(10^{-12} \mathrm{erg} \mathrm{cm}^{2} \mathrm{~s}^{-1}\right)\end{array}$ & $\begin{array}{c}L_{0.5-2 \mathrm{keV}} \\
\left(10^{44} \mathrm{erg} \mathrm{s}^{-1}\right)\end{array}$ & $\begin{array}{c}L_{2-10 \mathrm{keV}} \\
\left(10^{44} \mathrm{erg} \mathrm{s}^{-1}\right)\end{array}$ & $R_{\mathrm{S} / \mathrm{P}}^{0.5-2}$ & $R_{\mathrm{S} / \mathrm{P}}^{0.5-10}$ \\
\hline $0007+106$ & 3.7 & 7.2 & 0.87 & 1.4 & 0.160 & 0.072 \\
\hline $0050+124$ & 9.15 & 8.44 & 1.39 & 0.78 & 0.360 & 0.212 \\
\hline $0157+001$ & 1.33 & 0.93 & 1.05 & 0.71 & 0.169 & 0.126 \\
\hline $0804+761$ & 11.6 & 11.12 & 3.43 & 2.86 & 0.489 & 0.238 \\
\hline $0844+349$ & 6.9 & 5.5 & 0.81 & 0.55 & 0.954 & 0.444 \\
\hline $0947+396$ & 1.79 & 1.87 & 2.55 & 2.26 & 0.814 & 0.336 \\
\hline $0953+414$ & 3.79 & 3.21 & 7.77 & 5.41 & 0.836 & 0.379 \\
\hline $1001+054$ & 0.03 & 0.12 & 0.10 & 0.13 & - & - \\
\hline $1048+342$ & 1.24 & 1.46 & 1.07 & 1.10 & 0.712 & 0.277 \\
\hline $1100+772$ & 2.48 & 3.83 & 8.97 & 11.19 & 0.650 & 0.318 \\
\hline $1114+445$ & 0.62 & 2.3 & 2.65 & 1.45 & 1.75 & 0.724 \\
\hline $1115+080$ & 0.23 & 0.36 & 42.1 & 65.3 & - & - \\
\hline $1115+407$ & 2.13 & 1.27 & 1.67 & 0.85 & 0.787 & 0.427 \\
\hline $1116+215$ & 5.2 & 3.3 & 5.0 & 3.1 & 0.473 & 0.258 \\
\hline $1202+281$ & 2.68 & 3.72 & 2.22 & 2.68 & 0.646 & 0.226 \\
\hline $1206+459$ & 0.14 & 0.24 & 8.55 & 14.9 & - & - \\
\hline $1211+143$ & 3.0 & 3.1 & 0.61 & 0.50 & 1.825 & 0.572 \\
\hline $1216+069$ & 1.08 & 1.38 & 4.69 & 4.8 & 0.948 & 0.351 \\
\hline $1226+023$ & 43.81 & 78.74 & 31.75 & 51.05 & 0.310 & 0.103 \\
\hline $1244+026$ & 5.87 & 2.53 & 0.36 & 0.14 & 0.470 & 0.297 \\
\hline $1307+085$ & 0.94 & 2.01 & 0.63 & 1.19 & 0.443 & 0.118 \\
\hline $1309+355$ & 0.45 & 0.73 & 0.46 & 0.67 & 0.175 & 0.077 \\
\hline $1322+659$ & 2.25 & 1.33 & 2.21 & 1.08 & 0.650 & 0.367 \\
\hline $1352+183$ & 2.30 & 2.00 & 1.85 & 1.36 & 1.121 & 0.464 \\
\hline $1402+261$ & 3.0 & 1.9 & 2.7 & 1.4 & 0.851 & 0.427 \\
\hline $1404+226$ & 0.51 & 0.10 & 2.31 & 2.87 & 0.423 & 0.246 \\
\hline $1407+265$ & 0.94 & 0.80 & 53.31 & 41.20 & 0.105 & 0.058 \\
\hline $1411+442$ & 0.09 & 0.46 & 0.35 & 0.25 & 0.061 & 0.062 \\
\hline $1415+451$ & 1.4 & 1.1 & 0.5 & 0.4 & 0.696 & 0.331 \\
\hline $1427+480$ & 1.2 & 1.1 & 2.0 & 1.6 & 0.634 & 0.284 \\
\hline $1440+356$ & 5.9 & 3.9 & 1.0 & 0.58 & 2.432 & 1.076 \\
\hline $1444+407$ & 0.9 & 0.6 & 2.6 & 1.3 & 3.009 & 1.420 \\
\hline $1501+106$ & 17.2 & 16.2 & 0.57 & 0.49 & 0.761 & 0.311 \\
\hline $1512+370$ & 1.56 & 1.97 & 8.51 & 8.84 & 2.050 & 0.859 \\
\hline $1613+658$ & 2.77 & 4.15 & 1.40 & 1.78 & 0.327 & 0.121 \\
\hline $1626+554$ & 3.04 & 3.08 & 1.57 & 1.46 & 0.708 & 0.354 \\
\hline $1630+377$ & 0.14 & 0.15 & 31.1 & 20.9 & 1.570 & 0.774 \\
\hline $1634+706$ & 1.0 & 1.16 & 141.6 & 127.9 & 0.591 & 0.335 \\
\hline $2214+139$ & 0.31 & 3.26 & 0.39 & 0.48 & 0.168 & 0.111 \\
\hline $2302+029$ & 0.24 & 0.27 & 25.9 & 16.2 & 0.649 & 0.336 \\
\hline
\end{tabular}

This study reveals that none of the 20 pairs of parameters are significantly (i.e. $>99 \%$ ) correlated. Nevertheless, we found marginal hints of a non-trivial correlation for five of them, i.e. $k T_{\mathrm{BB}}-L_{0.5-2 \mathrm{keV}}, k T_{\mathrm{BB}}-L_{2-10 \mathrm{keV}}, k T_{\mathrm{Brem}}-L_{0.5-2 \mathrm{keV}}$, $k T_{\mathrm{Brem}}-L_{2-10 \mathrm{keV}}$ and $\Gamma_{\mathrm{Soft}}-R_{\mathrm{S} / \mathrm{P}}^{0.5-10}$. Some of these correlations appear to be driven by the higher luminosity QSOs, 
Table 12. Spearman-rank correlations among spectral parameters of the soft excess tested models and X-ray parameters (luminosity and $\left.\Gamma_{2-12}\right)$. The displayed values are $\left(r_{\mathrm{s}}, P_{\mathrm{s}}\right)$, where $r_{\mathrm{s}}$ is the rank correlation coefficient and $P_{\mathrm{s}}$ is the significance probability. The significant (i.e. $\left.>95 \%\right)$ correlations are marked in boldface (see text for details).

\begin{tabular}{ccccc}
\hline \hline & $k T_{\mathrm{BB}}$ & $k T_{\text {DiskBB }}$ & $k T_{\text {Brem }}$ & $\Gamma_{\text {Soft }}$ \\
\hline$L_{0.5-2 \mathrm{keV}}$ & $(0.46, \mathbf{9 7 . 9})$ & $(-0.26,82.4)$ & $(0.41, \mathbf{9 6 . 8})$ & $(-0.15,52.1)$ \\
$L_{2-10 \mathrm{keV}}$ & $(0.48, \mathbf{9 8 . 5})$ & $(-0.22,73.1)$ & $(0.44, \mathbf{9 8 . 2})$ & $(-0.23,73.7)$ \\
$R_{\mathrm{S} / \mathrm{P}}^{0.5-2}$ & $(0.04,13.3)$ & $(-0.09,35.1)$ & $(-0.07,29.0)$ & $(0.39,94.7)$ \\
$R_{\mathrm{S} / \mathrm{P}}^{0.5-10}$ & $(0.12,41.2)$ & $(-0.11,42.3)$ & $(-0.10,39.9)$ & $(0.45,97.4)$ \\
$\Gamma_{2-12}$ & $(0.26,78.1)$ & $(-0.20,70.8)$ & $(-0.07,27.6)$ & $(0.33,89.4)$ \\
\hline
\end{tabular}

Table 13. Spearman-rank correlations among X-ray observables and the radio-loudness parameter $R_{\mathrm{L}}$, the redshift, the $F W H M$ of the $\mathrm{H}_{\beta}$ emission line, the black hole mass $M_{\mathrm{BH}}$ and the accretion rate $\dot{m}$ with respect to the Eddington rate. The displayed values are $\left(r_{\mathrm{s}}, P_{\mathrm{s}}\right)$, where $r_{\mathrm{s}}$ is the rank correlation coefficient and $P_{\mathrm{s}}$ is the significance probability. The significant (i.e. $>95 \%$ ) correlations are marked in boldface (see text for details).

\begin{tabular}{ccccc}
\hline \hline & $L_{2-10 \mathrm{keV}}$ & $R_{\mathrm{S} / \mathrm{P}}^{0.5-10}$ & $\Gamma_{2-12}$ & $\Gamma_{\text {Soft }}$ \\
\hline$R_{\mathrm{L}}{ }^{(a)}$ & $(0.12,55.2)$ & $(-0.48,99.8)$ & $(-0.003,2.0)$ & $(-0.37,93.2)$ \\
$z$ & $(0.76,99.9)$ & $(0.24,85.2)$ & $(0.08,39.0)$ & $(-0.07,25.7)$ \\
$F W H M\left(\mathrm{H}_{\beta}\right)^{(b)}$ & $(0.50,99.7)$ & $(-0.12,51.1)$ & $(-0.62,99.9)$ & $(-0.54,98.6)$ \\
$M_{\mathrm{BH}}{ }^{(c)}$ & $(0.67,99.9)$ & $(-0.03,13.1)$ & $(-0.52,99.8)$ & $(-0.16,51.2)$ \\
$\dot{m}^{(d)}$ & $(-0.39,97.5)$ & $(-0.05,19.0)$ & $(0.41,97.9)$ & $(-0.09,30.1)$ \\
\hline
\end{tabular}

Data taken from: ${ }^{(a)}$ Kellermann et al. (1994); ${ }^{(b)}$ Boroson \& Green (1992); ${ }^{(c)}$ Gierlinski \& Done (2004), P04 and Shields et al. (2003); ${ }^{(d)}$ P04, Shields et al. (2003), and Woo \& Urry (2002).

$L_{2-10}>10^{45} \mathrm{erg} \mathrm{s}^{-1}$. For those relationships with $P_{\mathrm{s}}>95 \%$, we also performed a linear regression fit to the data which, contrary to the Spearman-rank correlation test, takes also into account the uncertainties associated to the measurements. The values of $\chi^{2}$ obtained for each fit revealed that the probability that the two quantities are linearly correlated is lower than $50 \%$.

\subsection{Correlations with general source properties}

We also investigated by the Spearman-rank test the possible correlations among the most representative X-ray observables inferred from our analysis (i.e. $L_{2-10 \mathrm{keV}}, R_{\mathrm{S} / \mathrm{P}}^{0.5-10}, \Gamma_{2-12}$ and $\left.\Gamma_{\text {Soft }}\right)$ and some QSO physical characteristics, such as the radioloudness parameter $R_{\mathrm{L}}$, the redshift, the $F W H M$ of the $\mathrm{H}_{\beta}$ emission line, the black hole mass $M_{\mathrm{BH}}$, and the accretion rate $\dot{m}$ relative to the Eddington limit ${ }^{3}$ (i.e. $\dot{m}=\dot{M} / \dot{M}_{\text {Edd }}$ ). The results of the correlation analysis are presented in Table 13. We report significant and strong anticorrelations between the spectral indices $\Gamma_{2-12}$ and $\Gamma_{\text {Soft }}$ and the $\mathrm{H}_{\beta} F W H M$, confirming these well-known relationships found for both QSO-like (Reeves \& Turner 2000; P04; Laor et al. 1997) and Seyfert-like AGNs (Brandt et al. 1997). As already obtained for a smaller sample of PG QSOs by P04, the hard band slope also appears to anticorrelate with the black hole mass and to correlate with the fractional accretion rate $\dot{m}$. The latter, furthermore, has been

\footnotetext{
3 The five high- $z$ QSOs: $1115+080,1206+459,1407+265$, $1630+377,2302+029$ as well as $2212+139$ were excluded from the last three correlation analysis since there are no optical data available in the literature. For the calculation of $\dot{m}$ we applied the Eq. (1) reported in $\mathrm{P} 04$.
}

found to be inversely linked with the $L_{2-10 \mathrm{keV}}$ with a significance of $P_{\mathrm{s}}=97.5 \%$, i.e. larger than in P04. PG QSOs with small $M_{\mathrm{BH}}$ tend to be less X-ray luminous. Laor (2000) pointed out that nearly all the PG RLQs with $M_{\mathrm{BH}}>10^{9} M_{\odot}$ are radio-loud and this could have caused a bias in our correlation analysis. The (anti-)correlations between $\left(\Gamma_{2-12}\right) L_{2-10 \mathrm{keV}}$ and $M_{\mathrm{BH}}$ still hold when RLQs are excluded. An interesting discovery yielded by the present analysis is the very significant $\left(P_{\mathrm{S}}>99 \%\right)$ anticorrelation between the radio-loudness $R_{\mathrm{L}}$ and $R_{\mathrm{S} / \mathrm{P}}^{0.5-10}$, i.e. the ratio between the strength of the soft excess and the high energy power law component in the $0.5-10 \mathrm{keV}$ band. This seems to indicate that the soft excess emission in RLQs is less prominent than in RQQs. However, a larger number of objects with $R_{\mathrm{L}}>10$ (i.e. radio-loud) is needed to confirm the robustness of this relationship. Finally, no correlation among the three X-ray observables and redshift was detected apart from the trivial case $\left(L_{2-10 \mathrm{keV}}, z\right)$ which is due to selection effects. Figure 6 shows the plots corresponding to the very significant (i.e. at $>99 \%$ confidence level) non-trivial correlations found in our analysis.

\section{Discussion}

\subsection{The Hard X-ray continuum}

In Sect. 3.1 we presented the results for the power law fit in the high energy $(2-12 \mathrm{keV})$ band for all the QSO. The $\chi^{2}$ analysis shows that the fits are acceptable, i.e. $\chi_{v}^{2}<1.2$, in all but four cases, i.e. $1411+442\left(\chi_{v}^{2}=1.66\right) ; 1440+356\left(\chi_{v}^{2}=1.66\right)$; $1630+377\left(\chi_{v}^{2}=1.70\right) ; 2214+139\left(\chi_{v}^{2}=1.83\right)$. Except for 

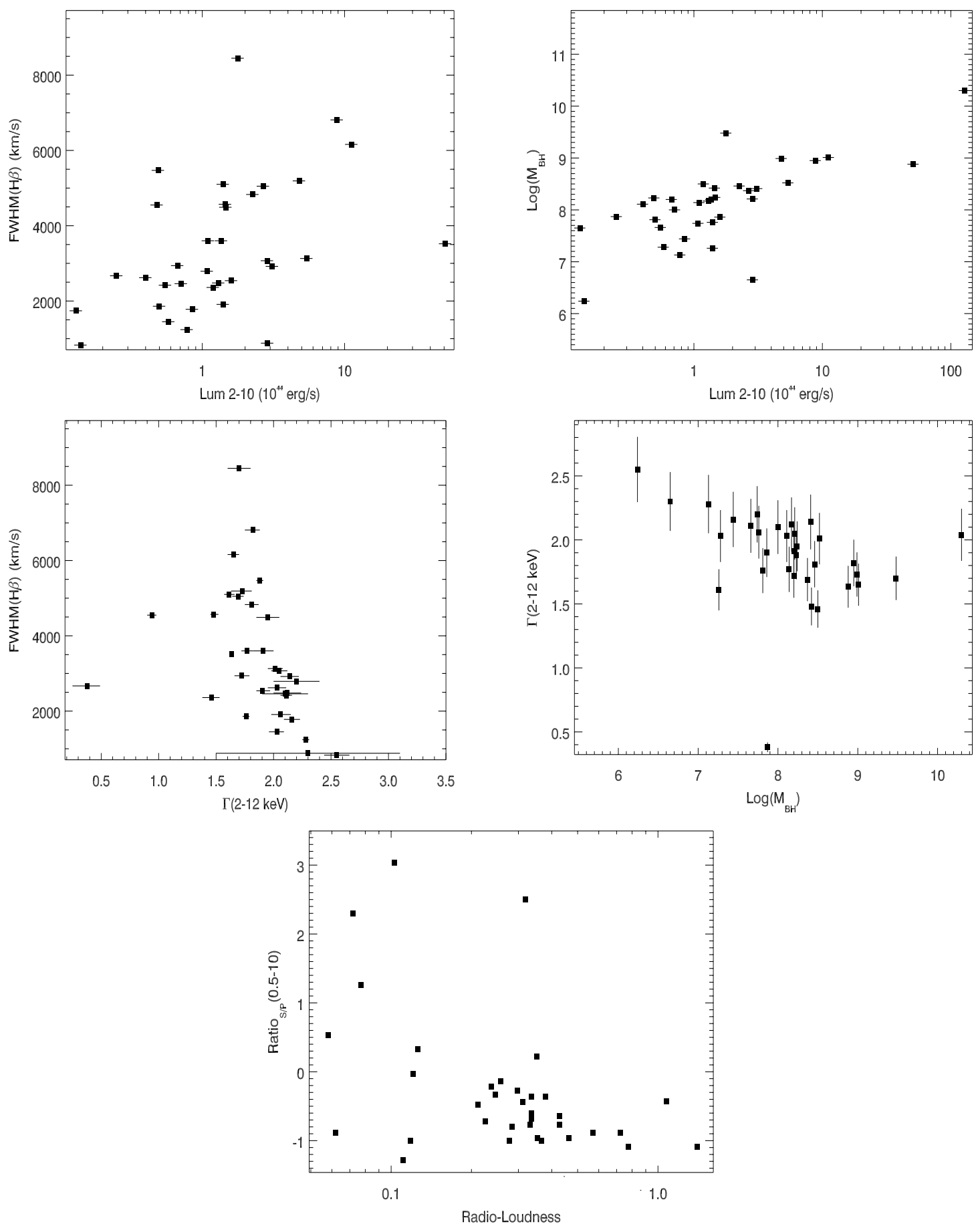

Fig. 6. Significant (i.e. at $>99 \%$ confidence level) correlations found among X-ray observables and QSO physical properties (See Table 13 and text for details). From top-left to bottom: a) $F W H M\left(\mathrm{H}_{\beta}\right)$ versus $L_{2-10 \mathrm{kev}}$; b) $M_{\mathrm{BH}}$ versus $L_{2-10 \mathrm{kev}}$; c) $F W H M\left(\mathrm{H}_{\beta}\right)$ versus $\Gamma_{2-12}$; d) $\Gamma_{2-12}$ versus $M_{\mathrm{BH}}$; e) $R_{\mathrm{S} / \mathrm{P}}^{0.5-10}$ versus $R_{\mathrm{L}}$.

$1630+377$, absorption was significantly detected in all these QSOs, being the most probable cause for the poor quality of the fit. In the case of $1630+377$, the presence of an intense iron line could be responsible for the large $\chi_{v}^{2}$.

The mean value found for the index of the power law was $\Gamma_{2-12 \mathrm{keV}}=1.87_{-0.10}^{+0.09}$ and shows a significant spread, $\sigma=$ $0.36_{-0.06}^{+0.08}$. The mean photon index is in good agreement with the results found from observations with previous satellites. Reeves \& Turner (2000) and George et al. (2000) reported an average $\Gamma_{2-10 \mathrm{keV}}=1.89 \pm 0.05$ and $\Gamma_{2-10 \mathrm{keV}}=1.97_{-0.09}^{+0.08}$ observing, respectively, 27 and 26 RQQs with ASCA (we compare $\Gamma_{2-12 \mathrm{keV}}$ with results from RQQ samples since RQQs represent $\sim 90 \%$ of our objects). A higher value (but consistent with ours within the statistical uncertainties) was derived from BeppoSAX data of 10 QSOs, i.e. $\Gamma_{2-10 \mathrm{keV}}=2.0 \pm 0.3$ (Mineo et al. 2000). It has been observed in several studies (Zamorani et al. 1981; Reeves \& Turner 2000) that RLQs have flatter spectral indices that RQQs. Our results confirm this 
trend with these mean values for the photon indices: $\Gamma_{\mathrm{RLQ}}=$ $1.63_{-0.01}^{+0.02}$ and $\Gamma_{\mathrm{RQQ}}=1.89 \pm 0.11$. The possible flatness of the spectral index for RLQs is consistent with a different process being responsible for the emission in the two types of QSO. While in RQQs the X-ray emission is thought to be associated with Compton up-scattering of soft photons originating in the accretion disk by hot electrons with a thermal distribution probably located in a corona above the accretion disk (Haardt \& Maraschi 1993; Reeves \& Turner 2000), in RLQs the effect of the relativistic jet could play an important role in the X-ray emission (Ghisellini et al. 1985; Reeves \& Turner 2000). Even though our results also show that the spectral index in RLQs is flatter than in RQQs, a study of a large sample of RLQs observed with ASCA (Sambruna et al. 1999) showed that if one compares RLQs and RQQs which match in X-ray luminosity, there is no clear evidence of a difference in the spectral slope of these two types of QSOs.

We note that our mean $\Gamma_{\text {soft }}=2.73_{-0.11}^{+0.12}$ (see Table 9) is similar to that found by Laor et al. (1997), i.e. $\Gamma_{\text {soft }}=2.62 \pm 0.09$. Therefore XMM-Newton EPIC results for the overall spectral shape of the QSOs appear to be consistent with those from ROSAT PSPC and ASCA GIS/SIS observations.

Finally, a very significant $\left(P_{\mathrm{s}}>99 \%\right)$ (anti-) correlation between $\left(\Gamma_{2-12 \mathrm{keV}}\right) L_{2-10 \mathrm{keV}}$ and the black hole mass was found (e.g. P04 and Sect. 3.7). Moreover, both these relationships still hold if only RQQs are considered. This points to the possibility that the black hole mass could play a major role in the definition of the hard X-ray properties in a QSO. Interestingly, a similar suggestion is reported by Laor (2000) who found a clear bimodality in the radio-loudness distribution as a function of the $M_{\mathrm{BH}}$ in the PG sample. These results can provide new insights in the ultimate driver of the AGN activity.

\subsection{The Soft excess}

The systematic modeling of the $0.3-12 \mathrm{keV}$ emission by different two-component continua presented in Sect. 3.2 allows us to draw useful conclusions on the physical nature of the soft excess.

Note the ubiquity of such a soft excess component: we detected a low-energy X-ray turn-up in 37 out of 40 QSOs ${ }^{4}$. Previous studies based on ASCA (George et al. 2000; Reeves \& Turner 2000) and BeppoSAX (Mineo et al. 2000) QSO samples reported the detection of an excess of emission with respect to the high energy power law below $1-1.5 \mathrm{keV}$ in $\sim 50-60 \%$ of the objects. However, as pointed out by these authors, one should bear in mind the limited statistics (in the case of some BeppoSAX exposures) and the limited bandpass $(0.6-10 \mathrm{keV}$ in the case of $A S C A$ ) affecting these observations.

All the models applied to account for the soft excess component (i.e. blackbody, multicolor blackbody, bremsstrahlung and a power law) provided a statistically acceptable description

\footnotetext{
4 For objects obscured by heavy absorption as 1411+442 and $2214+139$, however, the presence of the additional soft power law component can be more properly explained in terms of scattered emission from the absorbing medium and/or partial covering than a truly soft excess "emission".
}

of the broad-band X-ray spectrum for the majority of QSOs. In the following we discuss our results in the light of the physical scenario assumed in each tested model.

To first order approximation, the emission from a standard optically-thick accretion disk can be modeled by a blackbody or a multicolor blackbody components. The $\chi_{v}^{2}$ values obtained by the application of these two models reveal that these parameterizations give a fair representation of the EPIC QSO spectra (e.g. Tables 5 and 6). Nevertheless, the observed values of $k T_{\mathrm{BB}}$ and $k T_{\mathrm{MB}} \sim 0.15 \mathrm{keV}$ exceed the maximal temperature for an accretion disk (e.g. Frank et al. 1992, for a review) which is $k T_{\text {Max }} \sim 20-40 \mathrm{eV}$ for a black hole mass of $10^{7}-10^{8} M_{\odot}$ accreting at the Eddington rate. Furthermore, $k T_{\text {Max }}$ is an upper limit to the disk temperature since the accretion could be sub-Eddington and/or outer parts of the disk would likely be cooler. Hence, the mean values shown in Table 9 for the temperatures of a blackbody $\left(k T_{\mathrm{BB}}\right)$ and a multicolor blackbody $\left(k T_{\mathrm{MB}}\right)$ argue that these models must be considered just as phenomenological and not as physically consistent models. Such an "optically-thick" scenario is even more critical in those quasars with a double blackbody component as the best fit for the soft excess. In fact, they show a soft energy feature too broad to be fitted by a single temperature blackbody and, consequently, the second blackbody component usually has an unrealistic $k T_{\mathrm{BB}} \gtrsim 0.25 \mathrm{keV}$.

The soft X-ray emission of starburst regions (SBRs) is known to be dominated by the emission from a one- or multicomponent hot diffuse plasma at temperatures in the range of $k T \sim 0.1-1 \mathrm{keV}$ (Ptak et al. 1999; Read \& Ponman 2001; Strickland \& Stevens 2000). This thermal emission can be represented by a bremsstrahlung model. We found that this model is an appropriate fit $\left(\chi_{v}^{2}<1.2\right)$ to the soft excess emission of an important fraction of the objects in the sample (i.e. 28 out of 37 QSOs), and is the best fit model for 8 sources. The mean temperature for the bremsstrahlung model obtained for the 28 objects of our sample, $k T_{\mathrm{BS}}=0.38 \pm 0.03 \mathrm{keV}$, is compatible with typical values in SBRs. The diffuse gas associated to the soft X-ray emission of the SBRs typically has abundances in the range $0.05-0.3 Z_{\odot}$ (Ptak et al. 1999; Read \& Ponman 2001). However, the bremsstrahlung model does not include any emission lines and therefore it is not possible to measure the abundances of the diffuse gas and test if they are compatible with the typical ones measured in SBRs. In order to determine these abundances, we fitted the spectra of the eight sources for which the bremsstrahlung model is the best fit for their soft excess emission with the MEKAL model (Mewe et al. 1986) leaving the abundances as a free parameter. The resulting values of the abundances were in all cases consistent with zero with upper limits in the range $0.011-0.15 Z_{\odot}$. Except for two objects, $1634+706$ with $Z \leq 0.15 Z$ $\odot$ and $2303+029$ with $Z \leq 0.1 Z_{\odot}$, the abundances are therefore lower than the typical values observed in SBRs. Moreover, in the soft band, the X-ray luminosities of SBRs vary in the range of $10^{38-41} \mathrm{erg} \mathrm{s}^{-1}$ (Ptak et al. 1999; Read \& Ponman 2001) while the luminosities associated to the optically thin soft X-ray component for these 8 QSOs are in all cases above $8 \times 10^{43} \mathrm{erg} \mathrm{s}^{-1}$, and, therefore, two orders of magnitude larger than the typical SBRs values, even considering extreme cases such as Ultra-Luminous Infrared Galaxies 
(Franceschini et al. 2003). Hence, on the basis of the abundances and luminosities inferred for our QSOs, it is very unlikely that the bremsstrahlung emission could be associated to powerful emission from a starburst region.

Even if (at the moment) quite speculative, another possible physical interpretation for the bremsstrahlung model is the emission from a transition layer between the accretion disk and the corona, as proposed by Nayakshin et al. (2000). These authors indeed suggested that in the case of sources with SED dominated by the UV thermal disk emission (such as the present PG QSOs) bremsstrahlung emission from such a layer could significantly contribute to the soft X-ray emission in AGNs and also a plasma temperature of $\sim 0.4 \mathrm{keV}$ should be observed.

Alternatively, the bremsstrahlung model can be interpreted, in a similar way as the blackbody and multicolor blackbody models, as a purely mathematical parametrization of the QSO continuum (e.g. Fiore et al. 1995).

The power law is also, obviously, just a functional form to describe the true soft excess emission, without any underlying specific physical scenario. For six QSOs in the sample the power law model gave the best fit. Notably, two QSOs (i.e. $0804+761$ and $1440+356)$ show a particularly flat slope of the high energy power law $\left(\Gamma_{\text {hard }}=1.3 \pm 0.7\right.$ and $\Gamma_{\text {hard }}=1.2_{-0.2}^{+0.4}$, respectively, but note the large errors). Furthermore both $\Gamma_{\text {hard }}$ are very marginally (or not) consistent with the $\Gamma_{2-12}$ measured for these QSOs, suggesting the presence of a hard tail instead of a soft excess. Such a hard X-ray feature could also be related to a strong reflection component (or/and a peculiar variability behavior) which cannot be constrained due to limited EPIC bandpass. However, such a scenario seems to be unlikely for these QSOs because of the lack of a strong iron line in their spectra (not detected in 1440+356 and with an $E W \sim 100 \mathrm{eV}$ in 0804+76; see Paper II). Interestingly both these QSOs are classified as Narrow Line Quasars (Osterbrock \& Pogge 1985), a class of objects that usually show extreme flux variations, steep X-ray slopes and very strong soft excess extending up to $\sim 3 \mathrm{keV}$ in some cases (e.g. O'Brien et al. 2001). This also seems the case for $1440+356$ where such a spectral component dominates the broad band X-ray luminosity (e.g. Table 11).

Comptonization of thermal disk emission has been suggested as a likely physical origin of the soft excess in the $\mathrm{X}$-ray spectra of type 1 AGNs. The electron population responsible for the up-scattering is thought to have a lower temperature (typically from 0.1 to few $\mathrm{keVs}$ ) than the population producing the observed hard X-ray emission (approximatively a power law in the $2-10 \mathrm{keV}$ band) with a $k T_{\mathrm{e}} \gtrsim 80 \mathrm{keV}$ (Perola et al. 2002). The exact location of such an electron population is still an open issue: a warm skin on the disk surface (Rozanska 1999), a transition between a cold and a hot disk (Magdziarz et al. 1998), or a single hybrid non-thermal/thermal plasma (Vaughan et al. 2002 and reference therein) are the most viable candidates. Interestingly, this soft X-ray component would contain the bulk of the bolometric luminosity of the source.

According to this scenario, recent studies (P04; Gierliński $\&$ Done 2004) indeed modeled the soft X-ray excess of PG QSOs by a "cool" Compton-scattering medium. This fit successfully reproduces the QSO spectral shape. However, the typical value found for the electron temperature is $k T_{\mathrm{e}} \sim \mathrm{a}$ few hundred eVs, with a very small range of variation through the broad distribution in luminosity and black hole mass within the sample. As pointed out by different authors (Brinkmann et al. 2004b; Page et al. 2004b; P04) the major problem with the Comptonization fit is the limited bandpass of XMM-Newton that does not allow to adequately constrain either the blackbody emission from the disk or the exponential cut-off at high energy. So a plausible explanation for the observed narrow range of $k T_{\mathrm{e}}$ values is an observational bias due to the lack of sensitivity below $0.3 \mathrm{keV}$ in the EPIC data. Furthermore, as pointed out by $\mathrm{P} 04$, the inferred values of $k T_{\mathrm{e}}$ imply an extreme compactness of the Comptonizing region, i.e. 0.1 gravitational radii $\left(\sim 10^{12} \mathrm{~cm}\right)$. This size is difficult to explain even in a scenario where the hard X-ray continuum is thought to be produced in discrete magnetic flares (instead of an extended corona) above the accretion disk (e.g. Merloni \& Fabian 2002, for a review). Nevertheless, Comptonization seems the most realistic interpretation of the soft excess in QSOs. Unfortunately, the spectral capabilities of Chandra and XMM-Newton are not sufficient to disentangle the well-known degeneracy between the $k T_{\mathrm{e}}$ and the optical depth of the scattering plasma (e.g. Vaughan et al. 2002; Brinkmann et al. 2004b). EUV to very hard X-rays (i.e. a few hundred keV) broad band observations can help to solve this issue.

Gierliński \& Done (2004) suggested interpreting the soft excess in RQQs as an artifact of unmodeled relativistically smeared partially ionized absorption because strong $\mathrm{O}$ and $\mathrm{Fe}$ absorption features at $0.7-0.9 \mathrm{keV}$ can lead to an apparent upward curvature below these energies, mimicking the presence of a true soft excess component. This scenario predicts a very steep $(\Gamma \gg 2)$ intrinsic continuum slope in the hard X-ray band (i.e. $>10 \mathrm{keV}$ ) for RQQs. However, such large values of the intrinsic photon index (i.e. corrected for both reflection and absorption) have not been observed neither by BeppoSAX or Ginga observations (e.g. Mineo et al. 2000; and Lawson \& Turner 1997, respectively). Nonetheless, even if not in such a dramatic way, broad "warm" absorption features due to atomic transitions could have some effect on the estimate of the real slope of the primary continuum emission. More sensitive high-energy spectroscopic studies carried out in the near future with Astro-E2 or Constellation- $X$ will make a definitive test of this hypothesis possible.

On the basis of RGS observations Branduardi-Raymond et al. (2001) proposed to explain the observed soft excess in the Seyfert galaxies MKN 766 and MCG-6-30-15 in terms of strong relativistically broadened recombination $\mathrm{H}$-like emission lines of $\mathrm{O}, \mathrm{N}$ and $\mathrm{C}$. However, as pointed out by Pounds \& Reeves (2002), all the objects for which this interpretation seems to be feasible have a low X-ray luminosity (i.e. $L_{\mathrm{X}} \sim 10^{42} \mathrm{erg} \mathrm{s}^{-1}$ ) and their soft excess component emerges very sharply below $\sim 0.7 \mathrm{keV}$ from the extrapolation of the 2-10 keV power law. On the contrary, all the soft excesses observed in PG QSOs turn up smoothly below $\sim 2 \mathrm{keV}$. So this soft X-ray broad emission lines scenario seems to fail for the sources (which all have $L_{\mathrm{X}}>10^{43} \mathrm{erg} \mathrm{s}^{-1}$ ) in our sample.

Finally, the finding of an anticorrelation between $R_{\mathrm{L}}$ and $R_{\mathrm{S} / \mathrm{P}}^{0.5-10}$ (see Table 13) seems to support the hypothesis that 
the soft excess in radio-loud QSOs is due to an X-ray emission component originating in the jets.

\subsection{The intrinsic absorption}

\subsubsection{Cold absorber}

In order to study the presence of a cold absorber in QSOs, we have added a neutral absorption component to the different models tested. The analysis reveals that the majority of the spectra does not require this component, and that the equivalent hydrogen column is negligible in comparison with the Galactic one. Only three sources, all of them RQQs, show the presence of a cold absorber, i.e. $0050+124,1115+080$ and $1411+442$. The former two have columns of $N_{\mathrm{H}} \leq 10^{21} \mathrm{~cm}^{-2}$, likely associated to their host galaxies. The strongest absorption was found for $1411+442, N_{\mathrm{H}}=2.3 \pm 0.4 \times 10^{22} \mathrm{~cm}^{-2}$, although because of the low statistic of the data it is not possible to confirm if this feature originates from cold absorption or is indeed related to a warm absorber. The rarity of intrinsic neutral absorption observed here is in agreement with previous studies of optically-selected QSOs (Laor et al. 1997; Mineo et al. 2000) and, in general, with the findings of recent wideangle deep XMM-Newton surveys (e.g. Barcons et al. 2002; Piconcelli et al. 2003; Caccianiga et al. 2004) which reported a small fraction of broad line AGNs with a significant cold absorption component in excess of the Galactic value.

Therefore, we can conclude that for PG QSOs there is a good correlation between optical spectral type and X-ray absorption properties, in agreement with the predictions of the AGN Unification Models.

\subsubsection{Warm absorber}

Warm (i.e. partially ionized) absorbers in active galaxies are revealed by the presence of absorption edges in their soft $\mathrm{X}$-ray spectra (Pan et al. 1990). In particular, OVII $(0.739 \mathrm{keV})$ and OVIII $(0.871 \mathrm{keV})$ absorption edges were the typical signatures of such ionized gas detected in ROSAT and ASCA low-resolution observations. Notably, Reynolds (1997) found a warm absorber in $\approx 50 \%$ of the well studied Seyfert galaxies.

The study of this spectral component took a remarkable step forward owing to the higher resolution $(R>100)$ of the grating spectrometers on-board XMM-Newton and Chandra. $\mathrm{H}-$ and He-like absorption lines of cosmically abundant elements such as $\mathrm{C}, \mathrm{O}, \mathrm{Ne}, \mathrm{Mg}$ and $\mathrm{Si}$ are the most numerous and strongest features observed in the soft X-ray spectra of bright Seyfert 1 galaxies. The presence between 0.73 and $0.77 \mathrm{keV}$ of the unresolved transition array (UTA, e.g. Behar et al. 2001) of iron M-shell ions is also particularly common. The X-ray absorption lines are typically blueshifted by a few hundred $\mathrm{km} \mathrm{s}^{-1}$ and show complex profiles due to a wealth of kinematic components. The ionized material is therefore in an outflow and it generally shows multiple ionization states (Kaastra et al. 2000; Collinge et al. 2001; Piconcelli et al. 2004).

On the contrary, the properties of the warm absorber in QSOs are poorly known so far. Nonetheless, it is widely accepted that the presence of ionized absorbing gas in such high luminosity AGNs is not as common as in Seyfert galaxies. Laor et al. (1997) indeed detected it in $\leq 5 \%$ of the optically selected QSO population, and, on the basis of a large ASCA sample, Reeves \& Turner (2000) and George et al. (2000) both confirmed the rare occurrence in their spectra of opacity due to ionized gas along the line of sight. Bearing in mind the limited statistics, George et al. (2000) did not find, however, significant differences in the physical parameters of the warm absorber (i.e. ionization state and column density) in low and high luminosity AGNs.

The scenario emerging from our analysis is completely different. In fact, we significantly detect absorption features due to ionized gas in the spectra of 18 out 40 QSOs, i.e. the $45 \%$ of the sample. This fraction is similar to what is found for Seyfert galaxies. The detection rate of warm absorbers appears therefore to be independent of the X-ray luminosity. This confirms the recent result of P04 based on a smaller sample of 21 QSOs observed by XMM-Newton, and puts it on a sounder statistical ground.

As reported in Sect. 3.4 most of these absorption features are observed in the range $0.7-0.8 \mathrm{keV}$ : this suggests that they are likely due to the He-like $\mathrm{O}$ edge and/or the blend of M-shell Fe lines (UTA). In the case of the edges found in the highenergy portion of the spectrum around $7 \mathrm{keV}$ (as in $1115+080$, $1211+143$ and 1226+023), the energy of these features is still consistent with an origin in "neutral" material (i.e. FeI-V). So an alternative explanation in terms of reflection from the optically thick accretion disk cannot be ruled out. The edge detected at $\sim 9 \mathrm{keV}$ in $1115+08$ would imply an ionization state higher than FeXXVI, so the possible origin of this feature in an ionized outflow (with a velocity of $0.34 c$ to account for the observed blueshift) as proposed by Chartas et al. (2003) seems to be the most reasonable.

Because of the strength of the absorption features in their spectra, for 4 objects (i.e. $1001+054,1114+445,1404+226$ and 2214+139) we could apply the ABSORI model in the fit and, consequently, derive some physical parameters of the warm absorber in these sources (see Table 10). For 2214+139 and the BAL QSO 1001+054 the inferred column densities of the ionized gas are larger than $10^{23} \mathrm{~cm}^{-2}$, i.e. among the highest seen by XMM-Newton and Chandra so far. Even more interesting are the results we report for $1114+445$ and $2214+139$. Their $X M M$-Newton spectra indeed show for the first time evidence for a multi-zone warm absorber in both QSOs.

These findings agree with the recent results obtained for Seyfert galaxies which claim a multiphase outflowing plasma as origin of the warm absorber. Unfortunately, because of the EPIC spectral resolution, we cannot draw any conclusion on the ionization structure (i.e. discrete or continuous) of the ionized wind.

All the QSOs in our sample classified as "Soft X-ray Weak" QSOs (SXWQs) by Brandt et al. (2000) (namely 1001+054, $1411+442$ and $2214+139$ ) have been found to be heavily absorbed. This finding supports the hypothesis that the observed soft X-ray "weakness" of SXWQs is likely due to the presence of absorbing matter along the line of sight instead of an "intrinsic" weakness due to a different SED and/or emission/accretion mechanisms at work in SXWQs with respect 
to "normal" QSOs. Furthermore, all SXWQs analyzed here also show prominent absorption lines in the UV and in 2 out of 3 SXWQs the absorption occurs in an ionized gas ${ }^{5}$. These results support the idea of a possible physical connection between UV and X-ray absorbers (Crenshaw et al. 2003).

\section{Conclusions}

We have presented the results of the spectral analysis of 40 QSOs EPIC spectra observed by XMM-Newton. These objects represent $\sim 45 \%$ of the complete PG QSOs sample. The main findings of our study are:

- The hard X-ray continuum slope was found to be $\Gamma=$ $1.89 \pm 0.11$ and $\Gamma=1.63_{-0.01}^{+0.02}$ for the radio-quiet and radioloud QSOs in the sample, respectively. These values are consistent with previous X-ray observations of these objects and, in particular, we confirm a flatter slope in RLQs than RQQs. Such a difference could suggest the presence of an extra continuum contribution from the self-synchrotron Compton jet emission (Zamorani et al. 1981).

- The soft excess is an ubiquitous feature of the low energy X-ray spectrum of the optically selected QSOs. We systematically fitted the spectrum of each object with four different two-component models. None of the two-component models tested provides a satisfactory description for all the QSO spectra. This result suggests that the shape of the soft excess is not a universal QSO property. Spectral parameters inferred by these fits strongly indicate that all the applied models should be interpreted as phenomenological (and not physical) parameterizations of the observed spectral shape. Direct thermal emission from the accretion disk is ruled out on the basis of the very high temperature derived for this optically thick component. Emission from a thermal plasma in a starburst is also ruled out because of the very large X-ray luminosities observed in our QSOs. A double power law model also provides a good description of some QSO spectra, and corresponds to a double Comptonization scenario for the X-ray emission. However, as also suggested by other authors (e.g. Gierliński \& Done 2004; Brinkmann et al. 2004b) current X-ray observations cannot effectively constrain the physical parameters of the two plasmas because of the lack of sensitivity in the EUV and at a few hundred keVs where the emission peaks of the two component are expected.

- Significant evidence of cold absorption was detected in only three objects. Of these, two show column density $\left(N_{\mathrm{H}} \sim 10^{21} \mathrm{~cm}^{-2}\right)$ consistent with the contribution from the host galaxy. For $1411+442$ (with $N_{\mathrm{H}} \sim 10^{23} \mathrm{~cm}^{-2}$ ) there are strong indications that the obscuration occurs in an ionized medium (Brinkmann et al. 2004a). Our result therefore agrees with the prediction of the AGN Unified Models (as well as recent observational results from wide-angle X-ray surveys) on the relationship between optical classification and X-ray absorption properties.

5 There is also a strong suggestion of the presence of a warm absorber in 1411+442 (e.g. Sect. 3.4; Brinkmann et al. 2004a).
- About 50\% of the QSOs show warm absorber features in their spectra. This finding is in contrast with previous studies based on ASCA or BeppoSAX data, where warm absorbers appeared to be rare. The fraction we observed is similar to that inferred for Seyfert 1 galaxies. The existence of ionized gas along the line of sight is independent of the X-ray luminosity. For two QSOs with the highest $\mathrm{S} / \mathrm{N}$ ratio (i.e. $1114+445$ and $2214+139$ ) we also significantly detected a complex (two-phase) warm absorber (see Piconcelli et al. 2004).

- All the SXWQs in the sample show evidence of obscuration (occurring in an ionized medium in most of the cases). This finding suggests that their "weakness" is just a matter of absorption instead of an unusual shape of the spectral energy distribution of this class of QSOs.

- We performed correlation analysis between the spectral parameters of the soft excess-tested models and the X-ray luminosities (Sect. 3.6) as well as between the hard Xray observables (i.e. $L_{2-10 \mathrm{keV}}, R_{\mathrm{S} / \mathrm{P}}^{0.5-10}$ and $\Gamma_{2-12}, \Gamma_{\mathrm{Soft}}$ ) and some physical parameters (i.e. $R_{\mathrm{L}}, z, F W H M\left(\mathrm{H}_{\beta}\right)$ and $\dot{m}$ ) (see Sect. 3.7). We confirmed the anticorrelations between both photon indices and $F W H M\left(\mathrm{H}_{\beta}\right)$ as reported in previous papers (P04, Reeves \& Turner 2000). We also found that $\dot{m}$ is inversely correlated with the $L_{2-10 \mathrm{keV}}$ and that the black hole mass (anti-)correlates with $\left(\Gamma_{2-12}\right) L_{2-10 \mathrm{keV}}$. Furthermore, we discovered a very significant anti-correlation between $R_{\mathrm{L}}$ and $R_{\mathrm{S} / \mathrm{P}}^{0.5-10}$, i.e. the ratio between the strength of the soft excess and the high energy power law component in the $0.5-10 \mathrm{keV}$ band.

The XMM-Newton view of optically selected QSOs suggests that there are no significant changes in the spectral properties of these high luminosity AGNs compared with the low luminosity broad line Seyfert galaxies. See also Paper II for the properties of the fluorescent iron K-shell emission lines in the objects studied here.

Acknowledgements. The authors wish to thank the referee, Dr. James Reeves, for the prompt and encouraging report and the useful comments, which have significantly improved the paper. We also would to thank the XMM-Newton SOC science support team members at ESAC. This paper is based on observations obtained with $X M M-N e w t o n$, an ESA science mission with instruments and contributions directly funded by ESA Member States and the USA (NASA). This research has made use of the NASA/IPAC Extragalactic Database (NED) which is operated by the Jet Propulsion Laboratory, California Institute of Technology, under contract with the National Aeronautics and Space Administration.

\section{References}

Anders, E., \& Grevesse, N. 1989, Geochim. Cosmochim. Acta, 53, 197

Arnaud, K. A. 1996, Astronomical Data Analyis and Systems, ASP Conf. Ser., 101, 17

Ashton, C. E., Page, M. J., Blustin, A. J., et al. 2004, MNRAS, in press Barcons, X., Carrera, F. J., Watson, M. G., et al. 2002, A\&A, 382, 522 Behar, E., Sako, M., \& Kahn, S. M. 2001, ApJ, 563, 497 
Bennett, C. L., Halpern, M., Hinshaw, G., et al. 2003, ApJS, 148, 1 Brandt, W. N., Mathur, S., \& Elvis, M. 1997, MNRAS, 285, 25 Brandt, W. N., Laor, A., \& Wills, B. J. 2000, ApJ, 528, 637

Branduardi-Raymond, G., Sako, M., Kahn, S. M., et al. 2001, A\&A, 365,140

Brinkmann, W., Papadakis, I. E., \& Ferrero, E. 2004a, A\&A, 414, 107

Brinkmann, W., Arevalo, P., Gliozzi, M., \& Ferrero, E. 2004b, A\&A, 415, 959

Cappi, M., Matsuoka, M., Comastri, A., et al. 1997, ApJ, 478, 492

Caccianiga, A., Severgnini, P., Braito, V., et al. 2004, A\&A, 416, 901

Chartas, G., Brandt, W. N., \& Gallagher, S. C. 2003, ApJ, 595, 85

Collinge, M. J., Brandt, W. N., Kaspi, Shai, et al. 2001, ApJ, 557, 2

Comastri, A., Setti, G., Zamorani, G., et al. 1992, ApJ, 384, 62

Crenshaw, D. M., Kraemer, S. B., \& George, I. M. 2003, ARA\&A, 41, 117

Dickey, J. M., \& Lockman, F. J. 1990, ARA\&A, 28, 215

Done, C., Mulchaey, J. S., Mushotzky, R. F., \& Arnaud, K. A. 1992, ApJ, 395, 275

Elvis, M., Lockman, F., \& Wilkes, B. J. 1989, AJ, 97, 777

Fiore, F., Elvis, M., Siemiginowska, A., et al. 1995, ApJ, 449, 74

Franceschini, A., Braito, V., Persic, M., et al. 2003, MNRAS, 343, 1181

Frank, J., King, A., \& Raine, D. 1992, Accretion Power in Astrophysics (Cambridge University Press)

Gallo, L. C., Boller, T., Brandt, W. N., Fabian, A. C., \& Vaughan, S. 2004, A\&A, 417, 29

George, I. M., \& Fabian, A. C. 1991, MNRAS, 249, 352

George, I. M., Nandra, K., Laor, A., et al. 1997, ApJ, 491, 508

George, I. M., Turner, T. J., Yaqoob, T., et al. 2000, ApJ, 531, 52

Ghisellini, G., Maraschi, L., \& Treves, A. 1985, A\&A, 146, 204

Gierliński, M., \& Done, C. 2004, MNRAS, 349, L7

Haardt, F., \& Maraschi, L. 1991, ApJ, 380, 51

Kaastra, J. S., Mewe, R., Liedahl, D. A., Komossa, S., \& Brinkman, A. C. $2000, A \& A, 354,83$

Kellermann, K. I., Sramek, R., Schmidt, M., \& Green, R. F. 1994, AJ, 108,1163

Iwasawa, K., \& Taniguchi, Y. 1993, ApJ, 413, L15

Jimenez-Bailon, E., Piconcelli, E., Guainazzi, M., et al. 2004, A\&A, submitted (Paper II)

Laor, A. 2000, ApJ, 543, L111

Laor, A., Fiore, F., Elvis, M., Wilkes, B. J., \& McDowell, J. C. 1997, ApJ, 477, 93

Lawson, A. J., \& Turner, M. J. L. 1997, MNRAS, 288, 290

Lockman, F. J., \& Savage, B. D. 1995, ApJS, 97, 1

Loiseau, N. 2003, XMM-Newton Science Analysis Users Guide v2.1

Maccacaro, T., Gioia, I. M., Wolter, A., Zamorani, G., \& Stocke, J. T. 1988, ApJ, 326, 680

Magdziarz, P., Blaes, O. M., Zdziarski, A. A., Johnson, W. N., \& Smith, D. A. 1998, MNRAS, 301, 179

Mathur, S., Wilkes, B. J., \& Elvis, M. 1998, ApJ, 503, L23
Merloni, A., \& Fabian, A. C. 2002, Proc. of the Symposium on New Visions of the X-ray Universe in the XMM-Newton and Chandra Era, ESA-ESTEC [arXiv: astro-ph/0201531]

Mewe, R., Lemen, J. R., \& Van den Oord, G. H. J. 1986, A\&AS, 65 , 511

Mineo, T., Fiore, F., Laor, A., et al. 2000, A\&A, 359, 471

Monier, E. M., Mathur, S., Wilkes, B., \& Elvis, M. 2001, ApJ, 59, 675

Morrison, R., \& McCammon, D. 1983, ApJ, 270, 119

Murphy, E. D., Lockman, F., Laor, A., \& Elvis, M. 1996, ApJS, 105, 369

Nandra, K., George, I. M., Mushotzky, R. F., Turner, T. J., \& Yaqoob, T. 1997, ApJ, 488, L91

Nayakshin, S., Kazanas, D., \& Kallman, T. R. 2000, ApJ, 537, 833

O'Brien, P. T., Page, K., Reeves, J. N., et al. 2001, MNRAS, 327, 370

Osterbrock, D. E., \& Pogge, R. W. 1985, ApJ, 297, 166

Page, K. L., Turner, M. J. L., Done, C., et al. 2004a, MNRAS, 349, 57

Page, K. L., Schartel, N., Turner, M. J. L., \& O'Brien, P. T. 2004b, MNRAS, in press [arXiv: astro-ph/0404444]

Pan, H. C., Stewart, G. C., \& Pounds, K. A. 1990, MNRAS, 242, 177

Perola, G. C., Matt, G., \& Cappi, M. 2002, A\&A, 389, 802

Piconcelli, E., Cappi, M., Bassani, L., Di Cocco, G., \& Dadina, M. 2003, A\&A, 412, 689

Piconcelli, E., Jimenez-Bailon, E., Guainazzi, M., et al. 2004, MNRAS, 351, 161

Porquet, D., Reeves, J. N., O’Brien, P. T., \& Brinkmann, W. 2004, A\&A, 422, 85 (P04)

Pounds, K. A., \& Reeves, J. N. 2002, Proc. of New Visions of the X-ray Universe in the XMM-Newton and Chandra Era [arXiv: astro-ph/0201436]

Ptak, A., Serlemitsos, P., Yaqoob, T., \& Mushotzky, R. 1999, ApJS, 120,179

Read, A. M., \& Ponman, T. J. 2001, MNRAS, 328, 127

Reeves, J. N., \& Turner, M. J. L. 2000, MNRAS, 316, 234

Reynolds, C. S. 1997, MNRAS, 286, 513

Rozanska, A. 1999, MNRAS, 308, 751

Sambruna, R. M., Eracleous, M., \& Mushotzky, R. F. 1999, ApJ, 526, 60

Schartel, N., Schmidt, M., Fink, H. H., Hasinger, G., \& Truemper, J. 1997, A\&A, 320, 696

Schartel, N., et al. 2004, A\&A, submitted

Schmidt, M., \& Green, R. F. 1983, ApJ, 269, 352

Shields, G. A., Gebhardt, K., Salviander, S., et al. 2003, ApJ, 583, 124

Stark, A. A., Gammie, C. F., Wilson, R. W., et al. 1992, ApJS, 79, 77

Strickland, D. K., \& Stevens, I. R. 2000, MNRAS, 314, 511

Ulrich, M. H., Comastri, A., Komossa, S., \& Crane, P. 1999, A\&A, 350,816

Vaughan, S., Boller, Th., Fabian, A. C., et al. 2002, MNRAS, 337, 247

Woo, K. H., \& Urry, C. M. 2002, ApJ, 579, 530

Zamorani, G., Henry, J. P., Maccacaro, T., et al. 1981, ApJ, 245, 357 


\section{Online Material}


Table 2. Journal of observations.

\begin{tabular}{|c|c|c|c|c|c|c|c|c|c|c|c|}
\hline \multirow[t]{2}{*}{ PG name } & \multirow[t]{2}{*}{ Date } & \multirow[t]{2}{*}{ Rev. } & \multicolumn{3}{|c|}{ Exposure time (ks) } & \multirow[t]{2}{*}{ PG name } & \multirow[t]{2}{*}{ Date } & \multirow[t]{2}{*}{ Rev. } & \multicolumn{3}{|c|}{ Exposure time (ks) } \\
\hline & & & $p n$ & MOS1 & MOS2 & & & & $p n$ & MOS1 & MOS2 \\
\hline $0007+106$ & $2000-07-04$ & 104 & 9.9 & 7.5 & 7.5 & $1307+085$ & $2002-06-13$ & 460 & 11.2 & 13.3 & 13.3 \\
\hline $0050+124$ & $2002-06-22$ & 464 & 18.6 & 20.7 & 19.6 & $1309+355$ & 2002-06-10 & 458 & 25.2 & 28.4 & 27.3 \\
\hline $0157+001$ & $2000-07-29$ & 117 & 10.1 & 10.4 & 10.4 & $1322+659$ & $2002-05-11$ & 443 & 8.6 & 11.4 & 11.6 \\
\hline $0804+761$ & 2000-11-04 & 166 & 0.5 & 6.6 & 6.5 & $1352+183$ & 2002-07-20 & 478 & 12.3 & 14.7 & 14.9 \\
\hline $0844+349$ & 2000-11-04 & 166 & - & 23.3 & 23.3 & $1402+261$ & 2002-01-27 & 391 & 9.1 & 11.9 & 11.9 \\
\hline $0947+396$ & 2001-11-03 & 349 & 17.5 & 20.9 & 21.1 & $1404+226$ & 2001-06-18 & 279 & 16.3 & 19.4 & 18.1 \\
\hline $0953+414$ & 2001-11-22 & 358 & 11.5 & 12.4 & 14.7 & $1407+265$ & 2001-12-22 & 373 & 35.1 & 36.8 & 36.9 \\
\hline $1001+054$ & 2003-05-04 & 623 & 8.8 & 10.1 & 11.0 & $1411+442$ & $2002-07-10$ & 473 & 23.1 & 35.4 & 35.4 \\
\hline $1048+342$ & $2002-05-13$ & 444 & 28.1 & 32.1 & 32.1 & $1415+451$ & $2002-12-08$ & 549 & 21.1 & 24.1 & 24.1 \\
\hline $1100+772$ & 2002-11-01 & 530 & 19.2 & 22.5 & 22.5 & $1427+480$ & $2002-05-31$ & 453 & 35.2 & 38.9 & 38.5 \\
\hline $1114+445$ & $2002-05-14$ & 445 & 37.7 & 42.2 & 42.3 & $1440+356$ & $2001-12-23$ & 373 & - & 28.2 & 24.8 \\
\hline $1115+080$ & $2001-11-25$ & 360 & 53.8 & 61.8 & 61.8 & $1444+451$ & $2002-08-11$ & 489 & 18.5 & 21.0 & 21.0 \\
\hline $1115+407$ & 2002-05-17 & 446 & 15 & 20.2 & 19.9 & $1501+106$ & 2001-01-14 & 201 & 9.4 & - & - \\
\hline $1116+215$ & 2001-12-02 & 363 & 5.5 & 8.3 & 8.4 & $1512+370$ & $2002-08-25$ & 496 & 17.6 & 20.4 & 20.4 \\
\hline $1202+281$ & $2002-05-30$ & 453 & 12.9 & 17.3 & 17.3 & $1613+658$ & 2001-04-13 & 246 & 3.5 & 3.3 & 3.5 \\
\hline $1206+459$ & $2002-05-11$ & 443 & 9.1 & 12.2 & 12.2 & $1626+554$ & 2002-05-05 & 440 & 5.5 & 8.7 & 7.2 \\
\hline $1211+143$ & 2001-06-15 & 278 & 49.5 & 53.4 & 53.3 & $1630+377$ & 2002-01-06 & 380 & 12.3 & 15.7 & 16 \\
\hline $1216+069$ & 2002-12-18 & 554 & 14 & 16.5 & 16.5 & $1634+706$ & $2002-11-22$ & 541 & 15.7 & 19.0 & 18.8 \\
\hline $1226+023$ & $2000-06-15$ & 95 & 20.8 & - & - & $2214+139$ & $2002-05-12$ & 444 & 29 & 33.4 & 35.5 \\
\hline $1244+026$ & 2001-06-17 & 279 & - & 12.1 & 12.1 & $2302+029$ & 2001-11-29 & 362 & 9.0 & 12.4 & 12.2 \\
\hline
\end{tabular}

Table 3. Spectral fitting results. I: power law model applied to the hard band $(2-12 \mathrm{keV}) .1001+054$ is excluded due to the very poor statistics in this energy range.

\begin{tabular}{cccl|cccl}
\hline \hline PG name & $\Gamma_{2-12}$ & $\chi^{2}$ & d.o.f. & PG name & $\Gamma_{2-12}$ & $\chi^{2}$ & d.o.f. \\
\hline $0007+106$ & $1.61_{-0.04}^{+0.05}$ & 135 & 179 & $1307+085$ & $1.46_{-0.08}^{+0.07}$ & 117 & 92 \\
$0050+124$ & $2.28_{-0.03}^{+0.03}$ & 276 & 266 & $1309+355$ & $1.72_{-0.06}^{+0.07}$ & 95 & 87 \\
$0157+001$ & $2.1_{-0.2}^{+0.2}$ & 31 & 48 & $1322+659$ & $2.2_{-0.2}^{+0.2}$ & 91 & 88 \\
$0804+761$ & $2.05_{-0.07}^{+0.07}$ & 148 & 135 & $1352+183$ & $1.91_{-0.09}^{+0.09}$ & 126 & 97 \\
$0844+349$ & $2.11_{-0.05}^{+0.05}$ & 106 & 109 & $1402+261$ & $2.06_{-0.08}^{+0.09}$ & 77 & 92 \\
$0947+396$ & $1.81_{-0.06}^{+0.06}$ & 121 & 137 & $1404+226$ & $2.3_{-0.8}^{+0.8}$ & 5 & 9 \\
$0953+414$ & $2.01_{-0.06}^{+0.07}$ & 64 & 69 & $1407+265$ & $2.19_{-0.07}^{+0.07}$ & 83 & 118 \\
$1001+054$ & - & - & - & $1411+442$ & $0.38_{-0.13}^{+0.11}$ & 111 & 67 \\
$1048+342$ & $1.77_{-0.05}^{+0.05}$ & 113 & 149 & $1415+451$ & $2.03_{-0.08}^{+0.08}$ & 65 & 105 \\
$1100+772$ & $1.65_{-0.05}^{+0.05}$ & 180 & 220 & $1427+480$ & $1.90_{-0.05}^{+0.07}$ & 115 & 155 \\
$1114+445$ & $1.48_{-0.03}^{+0.04}$ & 255 & 241 & $1440+356$ & $2.03_{-0.07}^{+0.06}$ & 173 & 104 \\
$1115+080$ & $2.00_{-0.11}^{+0.11}$ & 87 & 87 & $1444+407$ & $2.12_{-0.12}^{+0.12}$ & 30 & 48 \\
$1115+407$ & $2.16_{-0.07}^{+0.07}$ & 94 & 100 & $1501+106$ & $1.88_{-0.03}^{+0.03}$ & 164 & 154 \\
$1116+215$ & $2.14_{-0.08}^{+0.08}$ & 105 & 106 & $1512+370$ & $1.82_{-0.07}^{+0.06}$ & 97 & 130 \\
$1202+281$ & $1.69_{-0.05}^{+0.05}$ & 189 & 195 & $1613+658$ & $1.70_{-0.10}^{+0.10}$ & 48 & 66 \\
$1206+459$ & $2.0_{-0.5}^{+0.4}$ & 15 & 12 & $1626+554$ & $1.95_{-0.10}^{+0.10}$ & 130 & 137 \\
$1211+143$ & $1.76_{-0.03}^{+0.03}$ & 380 & 278 & $1630+377$ & $2.2_{-0.4}^{+0.2}$ & 17 & 10 \\
$1216+069$ & $1.73_{-0.10}^{+0.08}$ & 54 & 79 & $1634+706$ & $2.04_{-0.09}^{+0.09}$ & 79 & 84 \\
$1226+023$ & $1.634_{-0.009}^{+0.009}$ & 260 & 193 & $2214+139$ & $0.94_{-0.04}^{+0.04}$ & 469 & 256 \\
$1244+026$ & $2.55_{-0.11}^{+0.11}$ & 69 & 55 & $2302+029$ & $2.2_{-0.2}^{+0.3}$ & 13 & 23 \\
\hline & & & & & & & \\
\hline
\end{tabular}


E. Piconcelli et al.: The XMM-Newton view of PG quasars. I., Online Material p 3

Table 4. Spectral fitting results. II: power law model (model PL) applied to the $0.3-12 \mathrm{keV}$ band.

\begin{tabular}{|c|c|c|c|c|c|c|c|c|c|}
\hline PG name & $\Gamma$ & $A_{\mathrm{pl}}$ & $\chi^{2}$ & d.o.f. & PG name & $\bar{\Gamma}$ & $A_{\mathrm{pl}}$ & $\overline{\chi^{2}}$ & d.o.f. \\
\hline $0007+106$ & $1.82_{-0.02}^{+0.01}$ & $2.32_{-0.02}^{+0.02} \times 10^{-2}$ & 407 & 307 & $1307+085$ & $1.76_{-0.02}^{+0.04}$ & $5.5_{-0.2}^{+0.2} \times 10^{-4}$ & 781 & 214 \\
\hline $0050+124$ & $1.77_{-0.02}^{+0.02}$ & $1.60_{-0.02}^{+0.02} \times 10^{-3}$ & 1359 & 394 & $1309+355$ & $1.94_{-0.03}^{+0.02}$ & $3.01_{-0.05}^{+0.05} \times 10^{-4}$ & 615 & 220 \\
\hline $0157+001$ & $2.50_{-0.03}^{+0.03}$ & $8.51_{-0.13}^{+0.13} \times 10^{-4}$ & 183 & 168 & $1322+659$ & $2.81_{-0.02}^{+0.02}$ & $1.64_{-0.01}^{+0.01} \times 10^{-3}$ & 515 & 216 \\
\hline $0804+761$ & $2.28_{-0.02}^{+0.01}$ & $7.21_{-0.07}^{+0.07} \times 10^{-3}$ & 364 & 266 & $1352+183$ & $2.50_{-0.02}^{+0.02}$ & $1.596_{-0.015}^{+0.015} \times 10^{-3}$ & 472 & 248 \\
\hline $0844+349$ & $2.41_{-0.01}^{+0.01}$ & $3.80_{-0.04}^{+0.04} \times 10^{-3}$ & 451 & 166 & $1402+261$ & $2.71_{-0.02}^{+0.02}$ & $2.10_{-0.02}^{+0.02} \times 10^{-3}$ & 449 & 219 \\
\hline $0947+396$ & $2.307_{-0.016}^{+0.016}$ & $1.341_{-0.012}^{+0.012} \times 10^{-3}$ & 496 & 265 & $1404+226$ & $4.18_{-0.05}^{+0.05}$ & $2.20_{-0.07}^{+0.07} \times 10^{-4}$ & 570 & 79 \\
\hline $0953+414$ & $2.46_{-0.01}^{+0.01}$ & $3.03_{-0.01}^{+0.01} \times 10^{-3}$ & 823 & 290 & $1407+265$ & $2.28_{-0.02}^{+0.02}$ & $2.00_{-0.03}^{+0.03} \times 10^{-3}$ & 243 & 246 \\
\hline $1001+054$ & $4.0_{-0.4}^{+0.4}$ & $1.7_{-0.2}^{+0.2} \times 10^{-5}$ & 156 & 10 & $1411+442$ & $2.6_{-0.1}^{+0.1}$ & $5.0_{-0.2}^{+0.2} \times 10^{-5}$ & 730 & 108 \\
\hline $1048+342$ & $2.24_{-0.02}^{+0.02}$ & $8.50_{-0.08}^{+0.08} \times 10^{-4}$ & 559 & 275 & +451 & $2.55_{-0.02}^{+0.02}$ & $8.49_{-0.08}^{+0.08} \times 10^{-4}$ & 458 & 232 \\
\hline $1100+772$ & $2.01_{-0.01}^{+0.01}$ & $2.16_{-0.02}^{+0.01} \times 10^{-3}$ & 606 & 348 & $1427+480$ & $2.36_{-0.014}^{+0.014}$ & $8.13_{-0.07}^{+0.07} \times 10^{-4}$ & 555 & 283 \\
\hline $1114+445$ & $1.14_{-0.01}^{+0.01}$ & $2.90_{-0.05}^{+0.05} \times 10^{-4}$ & 3607 & 368 & $1440+356$ & $2.65_{-0.02}^{+0.02}$ & $3.09_{-0.02}^{+0.03} \times 10^{-3}$ & 731 & 161 \\
\hline $1115+080$ & $1.77_{-0.02}^{+0.02}$ & $6.3_{-0.2}^{+0.2} \times 10^{-4}$ & 354 & 214 & $1444+407$ & $2.72_{-0.03}^{+0.03}$ & $8.14_{-0.10}^{+0.10} \times 10^{-4}$ & 357 & 168 \\
\hline $1115+407$ & $2.72_{-0.02}^{+0.02}$ & $1.49_{-0.02}^{+0.02} \times 10^{-3}$ & 530 & 234 & $1501+106$ & $2.47_{-0.02}^{+0.02}$ & $9.38_{-0.04}^{+0.04} \times 10^{-3}$ & 2281 & 225 \\
\hline $1116+215$ & $2.60_{-0.02}^{+0.02}$ & $3.45_{-0.03}^{+0.03} \times 10^{-3}$ & 456 & 234 & $1512+370$ & $2.15_{-0.02}^{+0.01}$ & $1.49_{-0.01}^{+0.01} \times 10^{-3}$ & 452 & 257 \\
\hline $1202+281$ & $1.69_{-0.05}^{+0.05}$ & $1.16_{-0.08}^{+0.08} \times 10^{-3}$ & 730 & 323 & $1613+658$ & $2.13_{-0.04}^{+0.04}$ & $1.82_{-0.04}^{+0.04} \times 10^{-3}$ & 354 & 184 \\
\hline $1206+459$ & $1.74_{-0.09}^{+0.09}$ & $2.4_{-0.2}^{+0.2} \times 10^{-4}$ & 40 & 42 & $1626+554$ & $2.25_{-0.02}^{+0.02}$ & $1.95_{-0.05}^{+0.05} \times 10^{-3}$ & 345 & 271 \\
\hline $1211+143$ & $3.14_{-0.02}^{+0.02}$ & $1.72_{-0.03}^{+0.02} \times 10^{-3}$ & 15193 & 404 & $1630+377$ & $2.25_{-0.07}^{+0.07}$ & $5.2_{-0.4}^{+0.4} \times 10^{-4}$ & 70 & 61 \\
\hline $1216+069$ & $2.19_{-0.03}^{+0.03}$ & $1.0_{-0.2}^{+0.1} \times 10^{-3}$ & 336 & 204 & $1634+706$ & $2.22_{-0.02}^{+0.08}$ & $3.57_{-0.09}^{+0.09} \times 10^{-3}$ & 238 & 210 \\
\hline $1226+023$ & $1.86_{-0.01}^{+0.01}$ & $2.85_{-0.01}^{+0.01} \times 10^{-2}$ & 6455 & 264 & $2214+139$ & $0.58_{-0.02}^{+0.02}$ & $1.34_{-0.03}^{+0.03} \times 10^{-4}$ & 2483 & 367 \\
\hline $1244+026$ & $2.90_{-0.02}^{+0.02}$ & $3.15_{-0.04}^{+0.04} \times 10^{-3}$ & 233 & 112 & $2302+029$ & $2.29_{-0.06}^{+0.06}$ & $6.7_{-0.4}^{+0.4} \times 10^{-4}$ & 94 & 68 \\
\hline
\end{tabular}

(†) Flux at $1 \mathrm{keV}$ in units of photons $/ \mathrm{keV} / \mathrm{cm}^{2} / \mathrm{s}$. 
E. Piconcelli et al.: The XMM-Newton view of PG quasars. I., Online Material $p 4$

Table 5. Spectral fitting results. III: power law plus blackbody model for the soft excess component (model A).

\begin{tabular}{|c|c|c|c|c|c|c|c|c|c|}
\hline PG Name & $A_{\mathrm{pl}}^{\dagger}$ & $\Gamma$ & $\begin{array}{c}A_{\mathrm{BB}}^{\dagger} \\
\left(\times 10^{-5}\right)\end{array}$ & $\begin{array}{c}k T \\
(\mathrm{keV})\end{array}$ & $\begin{array}{l}\text { Edge } \\
(\mathrm{keV})\end{array}$ & $\bar{\tau}$ & $\begin{array}{c}P_{\mathrm{F}-\text { test }} \\
(\%)\end{array}$ & $\chi_{v}^{2}$ & d.o.f. \\
\hline $0007+106$ & $1.97_{-0.07}^{+0.07} \times 10^{-3}$ & $1.67_{-0.02}^{+0.02}$ & $1.5_{-0.2}^{+0.2}$ & $0.16_{-0.09}^{+0.09}$ & - & - & - & 318 & 305 \\
\hline $0157+001$ & $7.1_{-0.5}^{+0.5} \times 10^{-4}$ & $2.24_{-0.07}^{+0.06}$ & $1.0_{-}^{=} 0.2$ & $0.127_{-0.010}^{+0.010}$ & - & - & - & 149 & 166 \\
\hline $0804+761$ & $6.6_{-0.1}^{+0.2} \times 10^{-3}$ & $2.18_{-0.04}^{+0.02}$ & $5.6_{-1.3}^{+1.6}$ & $0.112_{-0.013}^{+0.013}$ & - & - & - & 309 & 264 \\
\hline $0844+349$ & $3.31_{-0.08}^{+0.08} \times 10^{-3}$ & $2.24_{-0.03}^{+0.03}$ & $7.0_{-1}^{+2}$ & $0.108_{-0.008}^{+0.008}$ & - & - & - & 189 & 164 \\
\hline $0947+396$ & $1.01_{-0.05}^{+0.04} \times 10^{-3}$ & $1.99_{-0.04}^{+0.04}$ & $2.0_{-0.3}^{+0.2}$ & $0.160_{-0.008}^{+0.007}$ & $0.75_{-0.03}^{+0.02}$ & $0.28_{-0.08}^{+0.07}$ & $>99.9$ & 254 & 261 \\
\hline $0953+414$ & $2.21_{-0.07}^{+0.07} \times 10^{-3}$ & $2.12_{-0.04}^{+0.02}$ & $5.5_{-0.4}^{+0.4}$ & $0.16_{-0.04}^{+0.04}$ & $0.73_{-0.02}^{+0.02}$ & $0.33_{-0.05}^{+0.05}$ & $>99.9$ & 340 & 286 \\
\hline $1048+342$ & $6.5_{-0.2}^{+0.2} \times 10^{-4}$ & $1.93_{-0.03}^{+0.03}$ & $1.15_{-0.09}^{+0.09}$ & $0.133_{-0.05}^{+0.05}$ & - & - & - & 283 & 273 \\
\hline $1100+772$ & $1.83_{-0.05}^{+0.02} \times 10^{-3}$ & $1.84_{-0.02}^{+0.03}$ & $1.87_{-0.20}^{+0.20}$ & $0.141_{-0.010}^{+0.008}$ & - & - & - & 401 & 346 \\
\hline $1116+215$ & $2.8_{-0.1}^{+0.1} \times 10^{-4}$ & $2.29_{-0.03}^{+0.04}$ & $6.0_{-0.6}^{+0.5}$ & $0.113_{-0.006}^{+0.005}$ & - & - & - & 220 & 232 \\
\hline $1202+281$ & $1.37_{-0.05}^{+0.05} \times 10^{-3}$ & $1.80_{-0.03}^{+0.03}$ & $2.7_{-0.2}^{+0.2}$ & $0.159_{-0.007}^{+0.007}$ & $0.72_{-0.02}^{+0.02}$ & $0.33_{-0.07}^{+0.07}$ & $>99.9$ & 328 & 319 \\
\hline \multirow[t]{3}{*}{$1211+143$} & $9.6_{-0.2}^{+0.3} \times 10^{-4}$ & $1.715_{-0.012}^{+0.010}$ & $9.36_{-0.17}^{+0.12}$ & $0.1157_{-0.0015}^{+0.0013}$ & $0.771_{-0.005}^{+0.012}$ & $0.35_{-0.03}^{+0.04}$ & $>99.9$ & 771 & 400 \\
\hline & & & & & $0.953_{-0.008}^{+0.02}$ & $0.28_{-0.03}^{+0.03}$ & $>99.9$ & 571 & 398 \\
\hline & & & & & $7.28_{-0.12}^{+0.16}$ & $0.54_{-0.09}^{+0.09}$ & $>99.9$ & 458 & 396 \\
\hline $1216+069$ & $7.8_{-0.4}^{+0.3} \times 10^{-4}$ & $1.91_{-0.05}^{+0.05}$ & $1.4_{-0.2}^{+0.2}$ & $0.13_{-0.01}^{+0.01}$ & - & - & - & 181 & 202 \\
\hline $1244+026$ & $2.17_{-0.08}^{+0.09} \times 10^{-3}$ & $2.48_{-0.03}^{+0.03}$ & $4.93_{-0.04}^{+0.04}$ & $0.16_{-0.01}^{+0.01}$ & - & - & - & 125 & 110 \\
\hline $1307+085$ & $4.4_{-0.2}^{+0.2} \times 10^{-4}$ & $1.50_{-0.04}^{+0.04}$ & $1.2_{-0.1}^{+0.1}$ & $0.13_{-0.01}^{+0.01}$ & $0.74_{-0.02}^{+0.02}$ & $0.54_{-0.14}^{+0.10}$ & 99.9 & 232 & 210 \\
\hline $1309+355$ & $2.76_{-0.12}^{+0.12} \times 10^{-4}$ & $1.80_{-0.05}^{+0.04}$ & $0.53_{-0.10}^{+0.14}$ & $0.104_{-0.008}^{+0.008}$ & $0.74_{-0.02}^{+0.04}$ & $0.4_{-0.2}^{+0.2}$ & $>99.9$ & 238 & 216 \\
\hline $1402+261$ & $1.58_{-0.07}^{+0.08} \times 10^{-3}$ & $2.33_{-0.05}^{+0.05}$ & $4.2_{-0.4}^{+0.5}$ & $0.135_{-0.004}^{+0.003}$ & $0.68_{-0.05}^{+0.04}$ & $0.24_{-0.08}^{+0.08}$ & 99.8 & 243 & 215 \\
\hline $1407+265$ & $1.82_{-0.07}^{+0.07} \times 10^{-3}$ & $2.21_{-0.03}^{+0.03}$ & $0.7_{-0.3}^{+0.3}$ & $0.20_{-0.03}^{+0.03}$ & - & - & - & 223 & 244 \\
\hline \multirow[t]{2}{*}{$1415+451$} & $6.4_{-0.2}^{+0.3} \times 10^{-4}$ & $2.15_{-0.05}^{+0.05}$ & $1.7_{-0.2}^{+0.2}$ & $0.143_{-0.009}^{+0.008}$ & $0.78_{-0.04}^{+0.04}$ & $0.19_{-0.11}^{+0.09}$ & 99.7 & 196 & 228 \\
\hline & & & & & $0.62_{-0.03}^{+0.02}$ & $0.27_{-0.1}^{+0.09}$ & 97.9 & 189 & 226 \\
\hline $1427+480$ & $6.9_{-0.3}^{+0.3} \times 10^{-4}$ & $2.04_{-0.03}^{+0.03}$ & $1.16_{-0.10}^{+0.10}$ & $0.155_{-0.005}^{+0.007}$ & $0.72_{-0.02}^{+0.02}$ & $0.25_{-0.07}^{+0.06}$ & $>99.9$ & 269 & 279 \\
\hline $1512+370$ & $1.20_{-0.05}^{+0.05} \times 10^{-3}$ & $1.92_{-0.03}^{+0.03}$ & $1.7_{-0.2}^{+0.3}$ & $0.14_{-0.01}^{+0.01}$ & - & - & - & 258 & 255 \\
\hline $1613+658$ & $1.50_{-0.09}^{+0.09} \times 10^{-3}$ & $1.80_{-0.03}^{+0.07}$ & $3.1_{-0.4}^{+0.4}$ & $0.13_{-0.01}^{+0.01}$ & $0.73_{-0.02}^{+0.02}$ & $0.5_{-0.2}^{+0.1}$ & 99.9 & 148 & 180 \\
\hline $1626+554$ & $1.73_{-0.06}^{+0.06} \times 10^{-3}$ & $2.07_{-0.05}^{+0.05}$ & $2.2_{-0.4}^{+0.4}$ & $0.100_{-0.001}^{+0.001}$ & - & - & - & 270 & 268 \\
\hline $1630+377$ & $3.9_{-0.3}^{+0.8} \times 10^{-4}$ & $2.05_{-0.12}^{+0.06}$ & $0.7_{-0.3}^{+0.8}$ & $0.21_{-0.04}^{+0.04}$ & - & - & - & 55 & 59 \\
\hline $1634+706$ & $2.8_{-0.3}^{+0.3} \times 10^{-3}$ & $2.07_{-0.05}^{+0.02}$ & $2.1_{-0.5}^{+0.5}$ & $0.31_{-0.03}^{+0.03}$ & - & - & - & 209 & 208 \\
\hline $2302+029$ & $4.9_{-0.6}^{+0.7} \times 10^{-4}$ & $2.05_{-0.09}^{+0.09}$ & $1.2_{-0.3}^{+0.6}$ & $0.18_{-0.02}^{+0.02}$ & - & - & - & 59 & 66 \\
\hline
\end{tabular}

$\left(^{\dagger}\right)$ Flux at $1 \mathrm{keV}$ in units of photons $/ \mathrm{keV} / \mathrm{cm}^{2} / \mathrm{s}$. 
E. Piconcelli et al.: The XMM-Newton view of PG quasars. I., Online Material p 5

Table 6. Spectral fitting results. IV: power law plus multi-color blackbody model for the soft excess component (model B).

\begin{tabular}{|c|c|c|c|c|c|c|c|c|c|}
\hline PG Name & $\overline{A_{\mathrm{pl}}^{\dagger}}$ & $\bar{\Gamma}$ & $\overline{A_{\text {DiskBB }}}$ & $\begin{array}{c}k T \\
(\mathrm{keV})\end{array}$ & $\begin{array}{l}\text { Edge } \\
(\mathrm{keV})\end{array}$ & $\tau$ & $\begin{array}{c}P_{\mathrm{F}-\text { test }} \\
(\%)\end{array}$ & $\chi_{v}^{2}$ & d.o.f. \\
\hline $0007+106$ & $1.9_{-0.1}^{+0.1} \times 10^{-3}$ & $1.63_{-0.04}^{+0.04}$ & $29_{-8}^{+10}$ & $0.23_{-0.02}^{+0.02}$ & - & - & - & 318 & 305 \\
\hline $0157+001$ & $6.6_{-0.6}^{+0.7} \times 10^{-4}$ & $2.18_{-0.10}^{+0.04}$ & $50_{-20}^{+30}$ & $0.17_{-0.02}^{+0.02}$ & - & - & - & 149 & 166 \\
\hline $0804+761$ & $6.5_{-0.2}^{+0.2} \times 10^{-3}$ & $2.16_{-0.02}^{+0.04}$ & $916_{-508}^{+1363}$ & $0.14_{-0.02}^{+0.02}$ & - & - & - & 307 & 264 \\
\hline $0844+349$ & $3.29_{-0.08}^{+0.08} \times 10^{-3}$ & $2.23_{-0.04}^{+0.03}$ & $2355_{-1185}^{+2390}$ & $0.12_{-0.01}^{+0.01}$ & - & - & - & 189 & 164 \\
\hline $0947+396$ & $9.3_{-0.7}^{+0.5} \times 10^{-4}$ & $1.93_{-0.06}^{+0.04}$ & $69_{-14}^{+17}$ & $0.189_{-0.011}^{+0.013}$ & $0.77_{-0.05}^{+0.03}$ & $0.24_{-0.06}^{+0.08}$ & $>99.9$ & 241 & 261 \\
\hline $0953+414$ & $2.1_{-0.1}^{+0.1} \times 10^{-3}$ & $2.06_{-0.03}^{+0.03}$ & $259_{-40}^{+41}$ & $0.17_{-0.06}^{+0.08}$ & $0.74_{-0.02}^{+0.02}$ & $0.26_{-0.05}^{+0.05}$ & $>99.9$ & 320 & 286 \\
\hline $1048+342$ & $6.1_{-0.3}^{+0.3} \times 10^{-4}$ & $1.88_{-0.04}^{+0.04}$ & $81_{-15}^{+18}$ & $0.16_{-0.04}^{+0.08}$ & - & - & - & 270 & 273 \\
\hline $1100+772$ & $1.77_{-0.06}^{+0.06} \times 10^{-3}$ & $1.82_{-0.03}^{+0.03}$ & $135_{-38}^{+64}$ & $0.15_{-0.01}^{+0.01}$ & - & - & - & 398 & 346 \\
\hline $1115+407$ & $1.08_{-0.05}^{+0.03} \times 10^{-3}$ & $2.33_{-0.05}^{+0.04}$ & $268_{-52}^{+76}$ & $0.153_{-0.010}^{+0.008}$ & $0.67_{-0.04}^{+0.02}$ & $0.21_{-0.06}^{+0.06}$ & 99.99 & 249 & 230 \\
\hline $1116+215$ & $2.6_{-0.1}^{+0.1} \times 10^{-3}$ & $2.24_{-0.05}^{+0.05}$ & $600_{-200}^{+300}$ & $0.15_{-0.01}^{+0.01}$ & $0.72_{-0.04}^{+0.04}$ & $0.13_{-0.09}^{+0.08}$ & 96.7 & 208 & 230 \\
\hline $1202+281$ & $1.28_{-0.07}^{+0.06} \times 10^{-3}$ & $1.75_{-0.04}^{+0.03}$ & $80_{-20}^{+20}$ & $0.194_{-0.010}^{+0.013}$ & $0.73_{-0.02}^{+0.02}$ & $0.27_{-0.06}^{+0.07}$ & $>99.9$ & 312 & 319 \\
\hline \multirow[t]{3}{*}{$1211+143$} & $9.4_{-0.3}^{+0.3} \times 10^{-4}$ & $1.696_{-0.03}^{+0.012}$ & $900_{-100}^{+60}$ & $0.152_{-0.002}^{+0.004}$ & $0.772_{-0.003}^{+0.009}$ & $0.48_{-0.03}^{+0.05}$ & $>99.9$ & 1004 & 400 \\
\hline & & & & & $0.959_{-0.007}^{+0.009}$ & $0.45_{-0.04}^{+0.05}$ & $>99.9$ & 592 & 398 \\
\hline & & & & & $7.28_{-0.13}^{+0.14}$ & $0.54_{-0.09}^{+0.09}$ & $>99.9$ & 462 & 396 \\
\hline $1216+069$ & $7.5_{-0.4}^{+0.4} \times 10^{-4}$ & $1.89_{-0.03}^{+0.02}$ & $183_{-64}^{+114}$ & $0.13_{-0.01}^{+0.01}$ & - & - & - & 180 & 202 \\
\hline $1244+026$ & $2.07_{-0.07}^{+0.10} \times 10^{-3}$ & $2.44_{-0.03}^{+0.03}$ & $234_{-13}^{+18}$ & $0.19_{-0.05}^{+0.07}$ & - & - & - & 130 & 110 \\
\hline $1307+085$ & $4.3_{-0.3}^{+0.3} \times 10^{-4}$ & $1.48_{-0.04}^{+0.04}$ & $112_{-51}^{+74}$ & $0.15_{-0.02}^{+0.02}$ & $0.75_{-0.02}^{+0.02}$ & $0.5_{-0.2}^{+0.2}$ & 99.9 & 238 & 210 \\
\hline $1309+355$ & $2.7_{-0.1}^{+0.1} \times 10^{-4}$ & $1.79_{-0.04}^{+0.04}$ & $88_{-66}^{+254}$ & $0.13_{-0.03}^{+0.03}$ & $0.74_{-0.02}^{+0.03}$ & $0.5_{-0.2}^{+0.2}$ & 99.9 & 238 & 216 \\
\hline $1322+659$ & $1.15_{-0.06}^{+0.06} \times 10^{-3}$ & $2.32_{-0.05}^{+0.05}$ & $661_{-119}^{+155}$ & $0.132_{-0.006}^{+0.006}$ & - & - & - & 250 & 214 \\
\hline \multirow[t]{2}{*}{$1352+183$} & $9.8_{-1.0}^{+1.0} \times 10^{-4}$ & $1.97_{-0.08}^{+0.08}$ & $94_{-14}^{+20}$ & $0.203_{-0.012}^{+0.010}$ & $0.68_{-0.03}^{+0.02}$ & $0.38_{-0.10}^{+0.08}$ & 100 & 276 & 244 \\
\hline & & & & & $0.87_{-0.06}^{+0.08}$ & $0.17_{-0.07}^{+0.09}$ & 99.5 & 264 & 242 \\
\hline $1402+261$ & $1.46_{-0.11}^{+0.08} \times 10^{-3}$ & $2.26_{-0.07}^{+0.05}$ & $320_{-80}^{+80}$ & $0.159_{-0.009}^{+0.012}$ & $0.72_{-0.02}^{+0.04}$ & $0.20_{-0.07}^{+0.09}$ & $>99.9$ & 227 & 215 \\
\hline $1407+265$ & $1.80_{-0.09}^{+0.09} \times 10^{-3}$ & $2.20_{-0.04}^{+0.04}$ & $32_{-17}^{+43}$ & $0.14_{-0.03}^{+0.03}$ & - & - & - & 224 & 244 \\
\hline \multirow[t]{2}{*}{$1415+451$} & $5.9_{-0.4}^{+0.4} \times 10^{-4}$ & $2.09_{-0.06}^{+0.06}$ & $90_{-20}^{+30}$ & $0.176_{-0.014}^{+0.016}$ & $0.80_{-0.05}^{+0.05}$ & $0.15_{-0.09}^{+0.09}$ & 99.9 & 186 & 228 \\
\hline & & & & & $0.64_{-0.04}^{+0.03}$ & $0.21_{-0.09}^{+0.06}$ & 98.8 & 179 & 226 \\
\hline $1427+480$ & $6.5_{-0.4}^{+0.3} \times 10^{-4}$ & $2.00_{-0.05}^{+0.04}$ & $60_{-10}^{+10}$ & $0.155_{-0.006}^{+0.007}$ & $0.73_{-0.03}^{+0.03}$ & $0.19_{-0.06}^{+0.07}$ & $>99.9$ & 261 & 279 \\
\hline $1444+407$ & $5.5_{-0.4}^{+0.4} \times 10^{-4}$ & $2.26_{-0.07}^{+0.07}$ & $134_{-23}^{+23}$ & $0.16_{-0.01}^{+0.01}$ & $0.72_{-0.03}^{+0.03}$ & $0.28_{-0.11}^{+0.08}$ & 99.9 & 193 & 164 \\
\hline $1512+370$ & $1.15_{-0.06}^{+0.05} \times 10^{-3}$ & $1.89_{-0.04}^{+0.04}$ & $147_{-43}^{+72}$ & $0.142_{-0.006}^{+0.012}$ & - & - & - & 249 & 255 \\
\hline $1613+658$ & $1.5_{-0.1}^{+0.1} \times 10^{-3}$ & $1.78_{-0.07}^{+0.07}$ & $263_{-113}^{+305}$ & $0.15_{-0.02}^{+0.02}$ & $0.74_{-0.02}^{+0.02}$ & $0.4_{-0.1}^{+0.2}$ & 99.9 & 151 & 180 \\
\hline $1626+554$ & $1.71_{-0.07}^{+0.07} \times 10^{-3}$ & $2.06_{-0.06}^{+0.06}$ & $635_{-300}^{+691}$ & $0.12_{-0.02}^{+0.02}$ & - & - & - & 268 & 268 \\
\hline $1630+377$ & $3.8_{-0.8}^{+0.7} \times 10^{-4}$ & $2.0_{-0.1}^{+0.1}$ & $70_{-0.50}^{+750}$ & $0.10_{-0.02}^{+0.04}$ & - & - & - & 55 & 59 \\
\hline $1634+706$ & $2.5_{-0.4}^{+0.3} \times 10^{-3}$ & $2.03_{-0.07}^{+0.06}$ & $21_{-8}^{+13}$ & $0.19_{-0.02}^{+0.02}$ & - & - & - & 206 & 208 \\
\hline $2302+029$ & $4.9_{-0.7}^{+0.6} \times 10^{-4}$ & $2.05_{-0.11}^{+0.09}$ & $170_{-130}^{+500}$ & $0.11_{-0.02}^{+0.03}$ & - & - & - & 59 & 66 \\
\hline
\end{tabular}

( $)$ Flux at $1 \mathrm{keV}$ in units of photons $/ \mathrm{keV} / \mathrm{cm}^{2} / \mathrm{s}$. 
E. Piconcelli et al.: The XMM-Newton view of PG quasars. I., Online Material $p 6$

Table 7. Spectral fitting results. V: power law plus bremsstrahlung model for the soft excess (model C).

\begin{tabular}{|c|c|c|c|c|c|c|c|c|c|}
\hline PG name & $\overline{A_{\mathrm{pl}}^{\dagger}}$ & $\bar{\Gamma}$ & $\overline{A_{\mathrm{Brem}}^{\dagger}}$ & $\begin{array}{c}k T \\
(\mathrm{keV})\end{array}$ & $\begin{array}{l}\text { Edge } \\
(\mathrm{keV})\end{array}$ & $\bar{\tau}$ & $\begin{array}{c}P_{\mathrm{F}-\text { test }} \\
(\%)\end{array}$ & $\chi_{v}^{2}$ & $\begin{array}{l}\text { d.o.f. } \\
\text {. }\end{array}$ \\
\hline $0007+106$ & $1.6_{-0.2}^{+0.2} \times 10^{-3}$ & $1.56_{-0.08}^{+0.04}$ & $2.1_{-0.3}^{+0.3} \times 10^{-3}$ & $0.69_{-0.09}^{+0.09}$ & - & - & - & 321 & 305 \\
\hline $0157+001$ & $5.7_{-1.1}^{+1.1} \times 10^{-4}$ & $2.08_{-0.14}^{+0.16}$ & $1.4_{-0.3}^{+0.2} \times 10^{-3}$ & $0.44_{-0.09}^{+0.08}$ & - & - & - & 150 & 166 \\
\hline $0804+761$ & $6.3_{-0.5}^{+0.5} \times 10^{-3}$ & $2.13_{-0.04}^{+0.04}$ & $1.2_{-0.3}^{+0.6} \times 10^{-2}$ & $0.31_{-0.08}^{+0.08}$ & - & - & - & 305 & 264 \\
\hline $0844+349$ & $3.2_{-0.1}^{+0.1} \times 10^{-3}$ & $2.22_{-0.03}^{+0.03}$ & $3.0_{-1}^{+2} \times 10^{-2}$ & $0.20_{-0.01}^{+0.01}$ & - & - & - & 189 & 164 \\
\hline $0947+396$ & $7.9_{-1.3}^{+0.6} \times 10^{-4}$ & $1.84_{-0.11}^{+0.05}$ & $2.9_{-0.2}^{+0.2} \times 10^{-3}$ & $0.49_{-0.03}^{+0.07}$ & $0.79_{-0.04}^{+0.04}$ & $0.17_{-0.05}^{+0.08}$ & $>99.9$ & 229 & 261 \\
\hline $0953+414$ & $8.5_{-0.5}^{+0.5} \times 10^{-3}$ & $1.99_{-0.06}^{+0.04}$ & $1.8_{-0.1}^{+0.1} \times 10^{-3}$ & $0.42_{-0.03}^{+0.03}$ & $0.76_{-0.03}^{+0.03}$ & $0.18_{-0.05}^{+0.05}$ & $>99.9$ & 312 & 286 \\
\hline $1048+342$ & $5.4_{-0.4}^{+0.4} \times 10^{-4}$ & $1.79_{-0.05}^{+0.05}$ & $1.9_{-0.1}^{+0.1} \times 10^{-3}$ & $0.41_{-0.03}^{+0.03}$ & - & - & - & 259 & 273 \\
\hline $1100+772$ & $1.62_{-0.10}^{+0.09} \times 10^{-3}$ & $1.76_{-0.05}^{+0.04}$ & $3.0_{-0.3}^{+0.4} \times 10^{-3}$ & $0.45_{-0.06}^{+0.07}$ & - & - & - & 390 & 346 \\
\hline $1115+407$ & $9.7_{-0.9}^{+0.7} \times 10^{-4}$ & $2.25_{-0.05}^{+0.04}$ & $5.70_{-0.04}^{+0.04} \times 10^{-3}$ & $0.34_{-0.03}^{+0.03}$ & $0.69_{-0.04}^{+0.04}$ & $0.15_{-0.06}^{+0.07}$ & 99.9 & 239 & 230 \\
\hline $1116+215$ & $2.4_{-0.2}^{+0.2} \times 10^{-3}$ & $2.18_{-0.06}^{+0.06}$ & $1.2_{-0.1}^{+0.2} \times 10^{-2}$ & $0.33_{-0.04}^{+0.04}$ & $0.75_{-0.06}^{+0.05}$ & $0.11_{-0.06}^{+0.07}$ & 95.6 & 203 & 230 \\
\hline $1202+281$ & $1.13_{-0.09}^{+0.09} \times 10^{-3}$ & $1.68_{-0.05}^{+0.05}$ & $3.5_{-0.2}^{+0.2} \times 10^{-3}$ & $0.49_{-0.05}^{+0.05}$ & $0.74_{-0.03}^{+0.03}$ & $0.20_{-0.06}^{+0.06}$ & $>99.9$ & 306 & 319 \\
\hline $1216+069$ & $7.0_{-0.6}^{+0.6} \times 10^{-4}$ & $1.84_{-0.06}^{+0.06}$ & $2.7_{-0.4}^{+0.6} \times 10^{-3}$ & $0.34_{-0.05}^{+0.06}$ & - & - & - & 178 & 202 \\
\hline $1307+085$ & $4.2_{-0.3}^{+0.2} \times 10^{-4}$ & $1.46_{-0.07}^{+0.04}$ & $2.0_{-0.4}^{+0.4} \times 10^{-3}$ & $0.32_{-0.04}^{+0.08}$ & $0.77_{-0.03}^{+0.02}$ & $0.5_{-0.1}^{+0.1}$ & 99.9 & 244 & 210 \\
\hline $1309+355$ & $2.7_{-0.2}^{+0.2} \times 10^{-4}$ & $1.78_{-0.06}^{+0.04}$ & $9.8_{-3.2}^{+0.7} \times 10^{-4}$ & $0.28_{-0.07}^{+0.10}$ & $0.75_{-0.02}^{+0.02}$ & $0.5_{-0.2}^{+0.2}$ & $>99.99$ & 238 & 216 \\
\hline $1322+659$ & $1.02_{-0.07}^{+0.07} \times 10^{-3}$ & $2.24_{-0.06}^{+0.06}$ & $9.0_{-0.7}^{+0.8} \times 10^{-3}$ & $0.29_{-0.02}^{+0.02}$ & - & - & - & 242 & 214 \\
\hline $1352+183$ & $8.5_{-1.2}^{+1.0} \times 10^{-4}$ & $1.89_{-0.11}^{+0.08}$ & $4.7_{-0.3}^{+0.3} \times 10^{-3}$ & $0.45_{-0.03}^{+0.04}$ & $0.71_{-0.03}^{+0.02}$ & $0.26_{-0.06}^{+0.07}$ & $>99.9$ & 263 & 244 \\
\hline $1402+261$ & $1.22_{-0.15}^{+0.12} \times 10^{-3}$ & $2.14_{-0.09}^{+0.07}$ & $7.8_{-0.6}^{+0.5} \times 10^{-3}$ & $0.37_{-0.03}^{+0.04}$ & $0.75_{-0.04}^{+0.04}$ & $0.17_{-0.06}^{+0.07}$ & $>99.9$ & 211 & 215 \\
\hline $1407+265$ & $1.78_{-0.09}^{+0.09} \times 10^{-3}$ & $2.20_{-0.03}^{+0.03}$ & $1.1_{-0.4}^{+0.5} \times 10^{-3}$ & $0.5_{-0.1}^{+0.2}$ & - & - & - & 224 & 244 \\
\hline $1415+451$ & $5.5_{-0.5}^{+0.5} \times 10^{-4}$ & $2.03_{-0.07}^{+0.07}$ & $2.8_{-0.2}^{+0.3} \times 10^{-3}$ & $0.36_{-0.04}^{+0.04}$ & $0.73_{-0.05}^{+0.05}$ & $0.15_{-0.07}^{+0.07}$ & 99.9 & 179 & 228 \\
\hline $1427+480$ & $5.9_{-0.7}^{+0.3} \times 10^{-4}$ & $1.94_{-0.09}^{+0.04}$ & $2.06_{-0.14}^{+0.16} \times 10^{-3}$ & $0.43_{-0.03}^{+0.05}$ & $0.75_{-0.04}^{+0.04}$ & $0.12_{-0.04}^{+0.07}$ & $>99.9$ & 257 & 279 \\
\hline $1444+407$ & $5.2_{-0.8}^{+0.4} \times 10^{-4}$ & $2.23_{-0.14}^{+0.06}$ & $3.4_{-0.4}^{+0.4} \times 10^{-3}$ & $0.34_{-0.06}^{+0.06}$ & $0.72_{-0.03}^{+0.04}$ & $0.21_{-0.07}^{+0.11}$ & 99.9 & 191 & 164 \\
\hline $1501+106$ & $5.8_{-0.2}^{+0.2} \times 10^{-3}$ & $1.91_{-0.03}^{+0.03}$ & $3.4_{-0.1}^{+0.1} \times 10^{-2}$ & $0.34_{-0.02}^{+0.02}$ & $0.69_{-0.02}^{+0.02}$ & $0.17_{-0.03}^{+0.04}$ & $>99.9$ & 259 & 221 \\
\hline $1512+370$ & $1.05_{-0.07}^{+0.08} \times 10^{-3}$ & $1.84_{-0.05}^{+0.05}$ & $2.9_{-0.3}^{+0.5} \times 10^{-3}$ & $0.41_{-0.06}^{+0.04}$ & - & - & - & 239 & 255 \\
\hline $1613+658$ & $1.38_{-0.09}^{+0.08} \times 10^{-3}$ & $1.8_{-0.1}^{+0.1}$ & $5_{-1}^{+1} \times 10^{-3}$ & $0.33_{-0.07}^{+0.09}$ & $0.75_{-0.03}^{+0.02}$ & $0.4_{-0.1}^{+0.1}$ & 99.9 & 154 & 180 \\
\hline $1626+554$ & $1.67_{-0.10}^{+0.08} \times 10^{-3}$ & $2.04_{-0.05}^{+0.06}$ & $5.2_{-1.1}^{+2.2} \times 10^{-3}$ & $0.24_{-0.05}^{+0.05}$ & - & - & - & 267 & 268 \\
\hline $1630+377$ & $3.9_{-0.7}^{+0.7} \times 10^{-4}$ & $2.0_{-0.2}^{+0.1}$ & $2_{-1}^{+6} \times 10^{-3}$ & $0.40_{-0.2}^{+0.4}$ & - & - & - & 55 & 59 \\
\hline $1634+706$ & $2.1_{-0.4}^{+0.4} \times 10^{-3}$ & $1.95_{-0.11}^{+0.08}$ & $2.9_{-0.6}^{+0.6} \times 10^{-3}$ & $1.0_{-0.2}^{+0.2}$ & - & - & - & 203 & 208 \\
\hline $2302+029$ & $4.7_{-0.8}^{+0.7} \times 10^{-4}$ & $2.03_{-0.11}^{+0.06}$ & $2.8_{-1.3}^{+4} \times 10^{-3}$ & $0.37_{-0.11}^{+0.13}$ & - & - & - & 58 & 66 \\
\hline
\end{tabular}

$\left(^{\dagger}\right)$ Flux at $1 \mathrm{keV}$ in units of photons $/ \mathrm{keV} / \mathrm{cm}^{2} / \mathrm{s}$. 
E. Piconcelli et al.: The XMM-Newton view of PG quasars. I., Online Material $p 7$

Table 8. Spectral fitting results. VI: double power law (model D).

\begin{tabular}{|c|c|c|c|c|c|c|c|c|c|}
\hline PG Name & $\overline{A_{\text {hard }}^{\dagger}}$ & 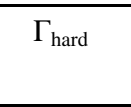 & $\overline{A_{\text {soft }}^{\dagger}}$ & $\overline{\Gamma_{\text {soft }}}$ & $\begin{array}{l}\text { Edge } \\
(\mathrm{keV})\end{array}$ & $\tau$ & $\begin{array}{c}P_{\mathrm{F}-\text { test }} \\
(\%)\end{array}$ & $\chi_{v}^{2}$ & d.o.f. \\
\hline $0007+106$ & $9_{-6}^{+3} \times 10^{-5}$ & $0.6_{-0.4}^{+0.7}$ & $2.2_{-0.3}^{+0.1} \times 10^{-3}$ & $1.95_{-0.05}^{+0.10}$ & - & - & - & 329 & 305 \\
\hline $0157+001$ & $5_{-4}^{+2} \times 10^{-5}$ & $1.1_{-0.8}^{+0.7}$ & $7.9_{-1.0}^{+0.5} \times 10^{-4}$ & $2.66_{-0.09}^{+0.20}$ & - & - & - & 151 & 166 \\
\hline $0804+761$ & $7_{-6}^{+25} \times 10^{-4}$ & $1.3_{-0.8}^{+0.6}$ & $6.5_{-2.6}^{+0.9} \times 10^{-3}$ & $2.5_{-0.1}^{+0.3}$ & - & - & - & 294 & 264 \\
\hline $0844+349$ & $2.9_{-0.4}^{+0.3} \times 10^{-3}$ & $2.13_{-0.09}^{+0.06}$ & $0.9_{-0.3}^{+0.5} \times 10^{-4}$ & $4.4_{-0.6}^{+0.7}$ & - & - & - & 184 & 164 \\
\hline $0947+396$ & $1.19_{-0.06}^{+0.10} \times 10^{-3}$ & $1.1_{-0.3}^{+0.2}$ & $1.19_{-0.10}^{+0.07} \times 10^{-3}$ & $2.58_{-0.07}^{+0.09}$ & - & - & - & 239 & 263 \\
\hline $1048+342$ & $1.1_{-0.5}^{+0.7} \times 10^{-4}$ & $1.1_{-0.2}^{+0.2}$ & $7.1_{-0.7}^{+0.4} \times 10^{-4}$ & $2.56_{-0.08}^{+0.10}$ & - & - & - & 273 & 273 \\
\hline $1100+772$ & $3_{-2}^{+3} \times 10^{-4}$ & $1.2_{-0.3}^{+0.2}$ & $1.8_{-0.3}^{+0.2} \times 10^{-3}$ & $2.3_{-0.1}^{+0.1}$ & - & - & - & 374 & 346 \\
\hline $1115+407$ & $1.30_{-0.08}^{+0.08} \times 10^{-3}$ & $1.3_{-0.4}^{+0.1}$ & $1.2_{-0.6}^{+0.9} \times 10^{-4}$ & $2.98_{-0.08}^{+0.07}$ & $8.1_{-0.3}^{+0.3}$ & $0.5_{-0.2}^{+0.4}$ & 99 & 239 & 230 \\
\hline $1116+215$ & $6_{-3}^{+5} \times 10^{-4}$ & $1.6_{-0.3}^{+0.2}$ & $2.7_{-0.5}^{+0.4} \times 10^{-3}$ & $2.9_{-0.1}^{+0.2}$ & $0.94_{-0.08}^{+0.08}$ & $0.07_{-0.04}^{+0.05}$ & 95 & 209 & 230 \\
\hline $1202+281$ & $6_{-2}^{+2} \times 10^{-4}$ & $1.42_{-0.2}^{+0.1}$ & $1.15_{-0.17}^{+0.3} \times 10^{-3}$ & $2.68_{-0.16}^{+0.12}$ & - & - & - & 331 & 321 \\
\hline $1216+069$ & $4_{-2}^{+1} \times 10^{-4}$ & $1.6_{-0.3}^{+0.1}$ & $5.9_{-1.5}^{+2.1} \times 10^{-4}$ & $2.9_{-0.3}^{+0.3}$ & - & - & - & 175 & 202 \\
\hline $1309+355$ & $2.0_{-0.9}^{+0.5} \times 10^{-4}$ & $1.7_{-0.2}^{+0.1}$ & $1.3_{-0.6}^{+1.1} \times 10^{-4}$ & $3.1_{-0.5}^{+0.6}$ & $0.76_{-0.02}^{+0.02}$ & $0.6_{-0.1}^{+0.1}$ & $>99.9$ & 238 & 216 \\
\hline $1352+183$ & $7_{-4}^{+8} \times 10^{-5}$ & $0.8_{-0.4}^{+0.4}$ & $1.52_{+0.10}^{+0.10} \times 10^{-3}$ & $2.68_{-0.05}^{+0.07}$ & $0.73_{-0.05}^{+0.05}$ & $0.09_{-0.05}^{+0.05}$ & 96 & 278 & 244 \\
\hline $1402+261$ & $1.7_{-0.7}^{+1.2} \times 10^{-4}$ & $1.3_{-0.2}^{+0.3}$ & $1.85_{-0.10}^{+0.09} \times 10^{-3}$ & $2.95_{-0.06}^{+0.08}$ & - & - & - & 216 & 217 \\
\hline $1407+265$ & $1.1_{-0.8}^{+0.5} \times 10^{-3}$ & $2.1_{-0.4}^{+1.2}$ & $9_{-7}^{+7} \times 10^{-4}$ & $2.7_{-1.0}^{+0.5}$ & - & - & - & 227 & 244 \\
\hline $1415+451$ & $1.6_{-0.6}^{+0.9} \times 10^{-4}$ & $1.5_{-0.2}^{+0.2}$ & $6.5_{-0.1}^{+0.6} \times 10^{-4}$ & $2.92_{-0.09}^{+0.1}$ & - & - & - & 199 & 230 \\
\hline $1427+480$ & $1.2_{-0.5}^{+0.8} \times 10^{-4}$ & $1.3_{-0.2}^{+0.2}$ & $17.6_{-0.8}^{+0.6} \times 10^{-4}$ & $2.67_{-0.08}^{+0.10}$ & - & - & - & 277 & 281 \\
\hline $1440+356$ & $3.0_{-1.0}^{+3} \times 10^{-4}$ & $1.2_{-0.2}^{+0.4}$ & $2.81_{-0.3}^{+0.11} \times 10^{-3}$ & $3.14_{-0.08}^{+0.2}$ & - & - & - & 191 & 158 \\
\hline $1444+407$ & $2_{-1}^{+1} \times 10^{-4}$ & $1.8_{-0.3}^{+0.3}$ & $5.7_{-0.1}^{+0.1} \times 10^{-4}$ & $3.3_{-0.3}^{+0.2}$ & - & - & - & 196 & 166 \\
\hline $1512+370$ & $5_{-3}^{+4} \times 10^{-4}$ & $1.6_{-0.3}^{+0.1}$ & $9_{-2}^{+3} \times 10^{-4}$ & $2.8_{-0.2}^{+0.2}$ & - & - & - & 236 & 255 \\
\hline $1613+658$ & $1.1_{-0.2}^{+0.2} \times 10^{-3}$ & $1.6_{-0.1}^{+0.1}$ & $5_{-2}^{+2} \times 10^{-4}$ & $3.7_{-0.4}^{+0.5}$ & $0.76_{-0.03}^{+0.03}$ & $0.4_{-0.1}^{+0.1}$ & $>99.9$ & 159 & 180 \\
\hline $1626+554$ & $1.1_{-0.7}^{+0.4} \times 10^{-3}$ & $1.9_{-0.4}^{+0.1}$ & $7_{-4}^{+7} \times 10^{-4}$ & $3.1_{-0.4}^{+0.70}$ & - & - & - & 266 & 268 \\
\hline $1630+377$ & $3_{-2}^{+1} \times 10^{-4}$ & $1.94_{-0.5}^{+0.2}$ & $3_{-2}^{+2} \times 10^{-4}$ & $3.7_{-2.3}^{+1.2}$ & - & - & - & 54 & 59 \\
\hline $1634+706$ & $8_{-7}^{+7} \times 10^{-6}$ & $0.3_{-0.1}^{+0.1}$ & $3.7_{-0.1}^{+0.1} \times 10^{-3}$ & $2.30_{-0.11}^{+0.09}$ & - & - & - & 203 & 208 \\
\hline $2302+029$ & $4.0_{-1.6}^{+1.3} \times 10^{-4}$ & $1.96_{-0.2}^{+0.16}$ & $3.9_{-1.6}^{+1.6} \times 10^{-4}$ & $4.1_{-0.8}^{+1.4}$ & - & - & - & 60 & 66 \\
\hline
\end{tabular}

(†) Flux at $1 \mathrm{keV}$ in units of photons $/ \mathrm{keV} / \mathrm{cm}^{2} / \mathrm{s}$ at $1 \mathrm{keV}$. 
Table 10. Spectral fitting results. VII: best fit model. A: bb; B: diskbb; C: brems; D: power law; E: double bb; F: absori; G: double absori + bb; H: partial-covering (cold)+raymond-smith; I: partial-covering(warm)+emission line; J: absori+bb; PL: simple power law. Models with * include an additional cold absorption component. All but one (i.e. model PL) models also include a power law component. For the results concerning the $\mathrm{Fe} \mathrm{K} \alpha$ line see Paper II.

\begin{tabular}{|c|c|c|c|c|c|c|c|c|}
\hline PG name & Model & $\bar{\Gamma}$ & $\begin{array}{c}k T / \Gamma_{\text {soft }} \\
(\mathrm{keV})\end{array}$ & $\begin{array}{c}k T \\
(\mathrm{keV})\end{array}$ & $\begin{array}{c}\xi /\left(N_{\mathrm{H}}\right) \\
(\mathrm{keV}) / 10^{22} \mathrm{~cm}^{-2}\end{array}$ & $\begin{array}{c}\text { Edge } / \tau \\
(\mathrm{keV})\end{array}$ & $\chi^{2}(v)$ & $\mathrm{Fe} \mathrm{K} \alpha$ \\
\hline $0007+106$ & $\mathrm{~A}$ & $1.71_{-0.04}^{+0.04}$ & $0.16_{-0.01}^{+0.01}$ & - & - & - & $312(302)$ & $\mathrm{Y}$ \\
\hline $0050+124$ & $\mathrm{E}^{*}$ & $2.31_{-0.03}^{+0.03}$ & $0.084_{-0.008}^{+0.008}$ & $0.19_{-0.02}^{+0.03}$ & $0.09_{-0.02}^{+0.02}$ & $0.647_{-0.009}^{+0.009} / 0.26_{-0.06}^{+0.06}$ & $523(383)$ & Y \\
\hline $0157+001$ & A & $2.24_{-0.07}^{+0.06}$ & $0.125_{-0.014}^{+0.012}$ & - & - & - & 149(166) & $\mathrm{N}$ \\
\hline $0804+761$ & $\mathrm{D}$ & $1.3_{-0.7}^{+0.7}$ & $2.5_{-0.1}^{+0.4}$ & - & - & - & $287(262)$ & $\mathrm{Y}$ \\
\hline $0844+349$ & $\mathrm{E}$ & $1.96_{-0.06}^{+0.09}$ & $0.112_{-0.004}^{+0.008}$ & $0.33_{-0.03}^{+0.03}$ & - & - & $162(162)$ & $\mathrm{N}$ \\
\hline $0947+396$ & $\mathrm{C}$ & $1.83_{-0.05}^{+0.07}$ & $0.50_{-0.04}^{+0.05}$ & - & - & $0.79_{-0.03}^{+0.04} / 0.18_{-0.04}^{+0.04}$ & $218(259)$ & $\mathrm{Y}$ \\
\hline $0953+414$ & $\mathrm{E}$ & $1.97_{-0.04}^{+0.04}$ & $0.119_{-0.009}^{+0.009}$ & $0.25_{-0.02}^{+0.02}$ & - & $0.73_{-0.03}^{+0.03} / 0.18_{-0.09}^{+0.06}$ & $308(284)$ & $\mathrm{N}$ \\
\hline $1001+054$ & $\mathrm{~F}$ & $2.0_{-0.1}^{+0.2}$ & - & - & $542_{-147}^{+97} / 19.2_{-7.3}^{+11.9}$ & - & $16(8)$ & $\mathrm{N}$ \\
\hline $1048+342$ & $\mathrm{E}$ & $1.74_{-0.08}^{+0.08}$ & $0.115_{-0.010}^{+0.008}$ & $0.27_{-0.02}^{+0.04}$ & - & - & $243(269)$ & Y \\
\hline $1100+772$ & $\mathrm{E}$ & $1.57_{-0.07}^{+0.07}$ & $0.133_{-0.007}^{+0.004}$ & $0.4_{-0.03}^{+0.03}$ & - & - & $339(342)$ & Y \\
\hline $1114+445$ & G & $1.85_{-0.03}^{+0.04}$ & $0.207_{-0.09}^{+0.09}$ & - & $89_{-30}^{+37} / 1.5_{-0.3}^{+0.4}$ & $4.0_{-0.5}^{+0.5} / 1.53_{-0.07}^{+0.12 a}$ & $315(360)$ & $\mathrm{Y}$ \\
\hline $1115+080$ & $\mathrm{PL}^{*}$ & $1.85_{-0.05}^{+0.05}$ & - & - & $0.33_{-0.07}^{+0.08}$ & $\begin{array}{l}7.1_{-0.4}^{+0.3} / 0.28_{-0.12}^{+0.13} \\
9.5_{-0.3}^{+0.3} / 0.31_{-0.15}^{+0.17}\end{array}$ & $\begin{array}{l}214(217) \\
204(215)\end{array}$ & $\mathrm{N}$ \\
\hline $1115+407$ & $\mathrm{E}$ & $2.18_{-0.08}^{+0.08}$ & $0.097_{-0.006}^{+0.006}$ & $0.238_{-0.003}^{+0.003}$ & - & - & $228(230)$ & Y \\
\hline $1116+215$ & $\mathrm{C}$ & $2.20_{-0.07}^{+0.06}$ & $0.32_{-0.04}^{+0.04}$ & - & - & $0.75_{-0.06}^{+0.05} / 0.10_{-0.06}^{+0.07}$ & 197(228) & $\mathrm{Y}$ \\
\hline $1202+281$ & $\mathrm{C}$ & $1.68_{-0.05}^{+0.05}$ & $0.49_{-0.05}^{+0.05}$ & - & - & $0.74_{-0.03}^{+0.03} / 0.20_{-0.06}^{+0.06}$ & $306(319)$ & $\mathrm{N}$ \\
\hline $1206+459$ & PL & $1.74_{-0.09}^{+0.09}$ & - & - & - & - & $40(42)$ & $\mathrm{N}$ \\
\hline $1211+143$ & $\mathrm{E}$ & $1.61_{-0.03}^{+0.05}$ & $0.113_{-0.004}^{+0.002}$ & $0.24_{-0.03}^{+0.02}$ & - & $\begin{array}{c}0.775_{-0.05}^{+0.11} / 0.39_{-0.04}^{+0.04} \\
0.967_{-0.009}^{+0.010} / 0.39_{-0.06}^{+0.04} \\
7.25_{-0.09}^{+0.14} / 0.66_{-0.07}^{+0.12}\end{array}$ & $402(392)$ & $\mathrm{Y}$ \\
\hline $1216+069$ & $\mathrm{E}$ & $1.67_{-0.09}^{+0.06}$ & $0.123_{-0.09}^{+0.07}$ & $0.349_{-0.05}^{+0.05}$ & - & - & $164(200)$ & $\mathrm{N}$ \\
\hline $1226+023$ & $\mathrm{E}$ & $1.60_{-0.01}^{+0.01}$ & $0.10_{-0.02}^{+0.02}$ & $0.24_{-0.01}^{+0.01}$ & - & $7.4_{-0.2}^{+0.1} / 0.10_{-0.03}^{+0.01}$ & $320(258)$ & $\mathrm{N}$ \\
\hline $1244+026$ & A & $2.48_{-0.03}^{+0.03}$ & $0.16_{-0.01}^{+0.01}$ & - & - & - & $125(110)$ & $\mathrm{N}$ \\
\hline $1307+085$ & A & $1.50_{-0.04}^{+0.04}$ & $0.13_{-0.01}^{+0.01}$ & - & - & $0.74_{-0.02}^{+0.02} / 0.54_{-0.14}^{+0.10}$ & $232(210)$ & $\mathrm{N}$ \\
\hline $1309+355$ & A & $1.81_{-0.04}^{+0.04}$ & $0.103_{-0.007}^{+0.011}$ & - & - & $0.75_{-0.03}^{+0.03} / 0.4_{-0.1}^{+0.2}$ & $225(214)$ & $\mathrm{Y}$ \\
\hline $1322+659$ & $\mathrm{C}$ & $2.25_{-0.08}^{+0.06}$ & $0.29_{-0.02}^{+0.01}$ & - & - & - & $235(212)$ & $\mathrm{Y}$ \\
\hline $1352+183$ & $\mathrm{E}$ & $1.84_{-0.10}^{+0.14}$ & $0.112_{-0.011}^{+0.012}$ & $0.26_{-0.03}^{+0.03}$ & - & $0.69_{-0.04}^{+0.03} / 0.21_{-0.11}^{+0.09}$ & $247(240)$ & $\mathrm{Y}$ \\
\hline $1402+261$ & $\mathrm{C}$ & $2.15_{-0.07}^{+0.08}$ & $0.37_{-0.03}^{+0.03}$ & - & - & $0.75_{-0.04}^{+0.04} / 0.17_{-0.06}^{+0.07}$ & $204(213)$ & $\mathrm{Y}$ \\
\hline $1404+226$ & $\mathrm{~J}$ & $2.3_{-0.4}^{+0.4}$ & $0.114_{-0.003}^{+0.002}$ & - & $44_{-26}^{+123} / 1.4_{-0.3}^{+0.6}$ & - & $60(75)$ & $\mathrm{N}$ \\
\hline $1407+265$ & A & $2.21_{-0.03}^{+0.03}$ & $0.20_{-0.03}^{+0.03}$ & - & - & - & $223(244)$ & $\mathrm{N}$ \\
\hline $1411+442$ & $\mathrm{H}$ & $2.3_{-0.2}^{+0.2}$ & $\equiv \Gamma$ & $0.15_{-0.03}^{+0.03}$ & $23.2_{-3.8}^{+3.8 c}$ & - & $82(102)$ & $\mathrm{Y}$ \\
\hline $1415+451$ & $\mathrm{E}$ & $2.00_{-0.10}^{+0.07}$ & $0.099_{-0.006}^{+0.006}$ & $0.24_{-0.03}^{+0.03}$ & - & - & $169(126)$ & $\mathrm{Y}$ \\
\hline $1427+480$ & $\mathrm{C}$ & $1.93_{-0.03}^{+0.06}$ & $0.44_{-0.04}^{+0.04}$ & - & - & $0.76_{-0.04}^{+0.03} / 0.12_{-0.04}^{+0.05}$ & $251(277)$ & $\mathrm{Y}$ \\
\hline $1440+356$ & $\mathrm{D}$ & $1.2_{-0.2}^{+0.4}$ & $3.14_{-0.08}^{+0.2}$ & - & - & - & 191(158) & $\mathrm{N}$ \\
\hline $1444+407$ & $\mathrm{D}$ & $1.8_{-0.3}^{+0.3}$ & $3.3_{-0.3}^{+0.2}$ & - & - & - & 196(166) & $\mathrm{N}$ \\
\hline $1501+106$ & $\mathrm{E}$ & $1.84_{-0.02}^{+0.02}$ & $0.0937_{-0.002}^{+0.0014}$ & $0.235_{-0.007}^{+0.005}$ & - & - & $240(221)$ & $\mathrm{N}$ \\
\hline $1512+370$ & $\mathrm{D}$ & $1.51_{-0.09}^{+0.09}$ & $2.71_{-0.15}^{+0.22}$ & - & - & - & $226(253)$ & $\mathrm{Y}$ \\
\hline $1613+658$ & A & $1.80_{-0.03}^{+0.07}$ & $0.13_{-0.01}^{+0.01}$ & - & - & $0.73_{-0.02}^{+0.02} / 0.5_{-0.2}^{+0.1}$ & $148(180)$ & $\mathrm{N}$ \\
\hline $1626+554$ & $\mathrm{D}$ & $1.9_{-0.4}^{+0.1}$ & $3.1_{-0.4}^{+0.7}$ & - & - & - & $266(268)$ & $\mathrm{N}$ \\
\hline $1630+377$ & $\mathrm{D}$ & $1.9_{-0.3}^{+0.3}$ & $3.4_{-1.1}^{+2.4}$ & - & - & - & $44(57)$ & $\mathrm{Y}$ \\
\hline $1634+706$ & $\mathrm{C}$ & $1.95_{-0.08}^{+0.11}$ & $1.1_{-0.2}^{+0.2}$ & - & - & - & $203(208)$ & $\mathrm{N}$ \\
\hline $2214+139$ & I & $1.88_{-0.04}^{+0.04}$ & $\equiv \Gamma$ & $0.57_{-0.01}^{+0.02 b}$ & $89_{-12}^{+14} / 8.9_{-0.5}^{+0.5}$ & $6_{-1}^{+3} / 1.7_{-0.4}^{+0.2 a}$ & $335(358)$ & $\mathrm{Y}$ \\
\hline $2302+029$ & $\mathrm{C}$ & $2.03_{-0.11}^{+0.06}$ & $0.37_{-0.11}^{+0.13}$ & - & - & - & $58(66)$ & $\mathrm{N}$ \\
\hline
\end{tabular}

${ }^{a}$ Values of $\xi$ and $N_{\mathrm{H}}$ of the second warm absorber component in $\mathrm{erg} / \mathrm{cm}^{2} / \mathrm{s}$ and $10^{22} \mathrm{~cm}^{-2}$, respectively; ${ }^{b}$ energy (in $\mathrm{keV}$ ) of the emission line (see text for details); ${ }^{c}$ value of $N_{\mathrm{H}} \times 10^{22} \mathrm{~cm}^{-2}$. 